\title{
Partner Selection in a Virtual Enterprise: A Group Multiattribute Decision Model with Weighted Possibilistic Mean Values
}

\author{
Fei Ye and Qiang Lin \\ School of Business Administration, South China University of Technology, Guangzhou 510640, China \\ Correspondence should be addressed to Fei Ye; yefei@scut.edu.cn
}

Received 2 October 2013; Accepted 25 November 2013

Academic Editor: Kim-Hua Tan

Copyright (C) 2013 F. Ye and Q. Lin. This is an open access article distributed under the Creative Commons Attribution License, which permits unrestricted use, distribution, and reproduction in any medium, provided the original work is properly cited.

\begin{abstract}
This paper proposes an extended technique for order preference by similarity to ideal solution (TOPSIS) for partner selection in a virtual enterprise (VE). The imprecise and fuzzy information of the partner candidate and the risk preferences of decision makers are both considered in the group multiattribute decision-making model. The weighted possibilistic mean values are used to handle triangular fuzzy numbers in the fuzzy environment. A ranking procedure for partner candidates is developed to help decision makers with varying risk preferences select the most suitable partners. Numerical examples are presented to reflect the feasibility and efficiency of the proposed TOPSIS. Results show that the varying risk preferences of decision makers play a significant role in the partner selection process in VE under a fuzzy environment.
\end{abstract}

\section{Introduction}

A virtual enterprise (VE) is a type of temporary alliance of independent, geographically dispersed organizations that aim to share skills and resources to exploit low-cost fastchanging market opportunities and achieve high-quality customer satisfaction [1-6]. VEs have become prevalent because of the increasing customer demands in the present global economy and the increasing complexity and diminishing life cycle of products. A VE is formed and dissolved by the appearance and disappearance of market opportunities, respectively.

A VE faces many important issues throughout its life cycle. Given the role of partners in the success of a VE, the proper selection of partners has received considerable research attention. Literature on partner selection can be divided into two categories.

The first literature category involves the information environment, wherein the verdicts of decision makers on candidate partners can be expressed in precise values. Various approaches have been proposed for VE partner selection. Talluri and Baker [7] proposed a two-phase mathematics programming method. Wu et al. [8] developed an integerprogramming method to minimize the transportation cost by geographic position and transportation approach. Ip et al. [1] described a risk-based partner selection problem and developed a mathematical programming model. Jarimo and Pulkkinen [9] presented a mixed-integer linear programming model to configure the virtual organization. Zhao et al. [10] developed a nonlinear integer-programming model to solve VE partner selection problems with precedence and due date constraints. $\mathrm{Ng}$ [11] proposed a weighted linear program to solve the multicriteria supplier selection problem. Other researchers simultaneously considered several factors such as cost, quality, credit, time, and risk for VE partner selection, thus making the partner selection problem for VE a type of multiattribute decision-making (MADM) problem. MADM methods, such as analytic hierarchy process (AHP), data envelopment analysis (DEA), and neural networks (NNs), have been developed to solve problems in VE partner selection. Sha and Che [12] developed a partner selection model based on AHP, multiattribute utility theory, and integer programming (IP) for multicriteria virtual integration; Sari et al. [13] applied the NN method to assess the performance 
of a particular partner in a VE. Wu [14] combined DEA, decision trees, and NNs to assess supplier performance. Liou [15] proposed a hybrid model to help airline companies select suitable partners for strategic alliances.

In reality, the decision makers are generally unsure of their judgement on candidates because the information about these candidates is uncertain and vague [4]. Thus, the second literature category considers the real-life vagueness and uncertainty of the partner selection process. Such problems can only be resolved by fuzzy set theory. Mikhailov [16] developed a fuzzy preference programming method for VE partner selection. Kahraman et al. [17] applied fuzzy AHP to select the supplier that highly satisfies the determined criteria. Wang and Lin [18] developed a fuzzy hybrid decision-aid model to select the best partner. Golec and Kahya [19] proposed a fuzzy model for competency-based employee evaluation and selection. Guneri et al. [20] presented an integrated fuzzy and linear programming approach for selecting suppliers and presented a set of linguistic values that are expressed in trapezoidal fuzzy numbers to assess the weights and ratings of the supplier selection criteria. Moreover, Crispim and Soua [3, 21] applied a fuzzy TOPSIS algorithm to rank alternative VE configurations. Ye and Li [4] proposed two MADM models with interval values to solve partner selection problems with partial information. Ye [5] proposed an extended TOPSIS method with interval-valued intuitionistic fuzzy numbers. Tseng [22] used linguistic preferences to describe the weights of green supply chain criteria and applied grey and fuzzy set theories to rank alternatives. Shaw et al. [23] used fuzzy AHP and fuzzy multiobjective linear programming to select the suitable supplier for the development of a low carbon supply chain. Liao and Kao [24] proposed an integrated fuzzy TOPSIS and multichoice goal programming approach to select suppliers in a supply chain.

However, these previous studies did not consider the risk preferences of decision makers in their proposed methods. The existence of contradicting methods in practice reflects the varying risk preference and aversion to risk neutrality of managers, particularly when they are placed under an uncertain environment. Given that risk-averse and risk-prone decision makers behave differently under similar situations, the risk preferences of decision makers must be considered when setting applicable and tailored decisions.

This paper uses fuzzy set theory to solve the vague and uncertain problems in the partner selection process. In contrast to the studies in the second literature category, we consider the risk preferences of decision makers to develop a partner selection approach that is consistent with actual practices. An extended TOPSIS is proposed in this study. The proposed extended TOPSIS considers the imprecise and uncertain information of partner candidates and the risk preferences of decision makers in the group MADM model. Weighted possibilistic mean values are used to manage triangular fuzzy numbers and establish the proposed TOPSIS model.

The rest of the paper is organized as follows. Section 2 introduces the basic concepts on the possibilistic mean values of fuzzy numbers. Section 3 presents the group MADM problem with triangular fuzzy numbers. Section 4 outlines the development of the extended TOPSIS. Section 5 presents an illustrative example. Section 6 summarizes the paper.

\section{Possibilistic Mean Values of Fuzzy Numbers}

In this section, we introduce some basic concepts related to possibilistic mean values of fuzzy numbers. First, we assume that a fuzzy number $A$ is a fuzzy set of the real line $\xi$ with a normal, fuzzy convex, and continuous membership function of bounded support. The family of fuzzy numbers can be denoted by $F(\xi)$. A $\gamma$-level set of a fuzzy number $A$ is defined by $[A]^{\gamma}=\{t \in \xi A(t) \geq \gamma\}$, as $\gamma>0$, and $[A]^{\gamma}=$ $\operatorname{cl}\{t \in \xi \mid A(t)>0\}$ (the closure of the support of $A$ ), as $\gamma=$ $0[25,26]$.

Definition 1 (see $[25,26]$ ). Let $A \in \xi$ be a fuzzy number with $[A]^{\gamma}=\left[a_{1}(\gamma), a_{2}(\gamma)\right], \gamma \in[0,1]$. The lower and upper possibilistic mean values of fuzzy number $A$ with $\gamma$-level set can be defined as $M_{*}(A)=2 \int_{0}^{1} \gamma a_{1}(\gamma) d \gamma$ and $M^{*}(A)=$ $2 \int_{0}^{1} \gamma a_{2}(\gamma) d \gamma$.

Definition 2 (see [25]). The interval-valued possibilistic mean of $A$ can be defined as $M(A)=\left[M_{*}(A), M^{*}(A)\right]$. However, the crisp possibilistic mean value of $A$ is defined as $\bar{M}(A)=$ $\left(M_{*}(A)+M^{*}(A)\right) / 2$.

According to Definition 2, Zhang et al. [26] defined the weighted possibilistic mean of fuzzy number $A$ as

$$
\bar{M}(A ; t)=(1-t) M_{*}(A)+t M^{*}(A), \quad t \in[0,1] .
$$

If $t=0$, then $\bar{M}(A ; 0)=M_{*}(A)$; if $t=1$, then $\bar{M}(A ; 1)$ $=M^{*}(A)$; if $t=1 / 2$, then $\bar{M}(A ; 1 / 2)=\left(M^{*}(A)+M_{*}(A)\right) / 2$. Obviously, for different $t$, we can give different importance to the lower and upper possibilistic mean values.

Researchers have focused on the computing of different approximations of fuzzy numbers, within which triangular fuzzy numbers and trapezoidal fuzzy numbers are the most popular fuzzy analysis methods. In this paper, we use triangular fuzzy numbers to express the judgments of decision makers on the information of the candidates. Let $A$ be a triangular fuzzy number with center $a$, left width $\alpha>0$, and right width $\beta>0$; that is, $A=(a-\alpha, a, a+\beta)$. According to Definition 2, a $\gamma$-level set of the fuzzy number $A$ can easily be computed as

$$
[A]^{\gamma}=[a-(1-\gamma) \alpha, a+(1-\gamma) \beta], \quad \forall \gamma \in[0,1] .
$$

According to Definition 1, we can get

$$
\begin{aligned}
& M_{*}(A)=2 \int_{0}^{1} \gamma(a-(1-\gamma) \alpha) d \gamma=a-\frac{\alpha}{3}, \\
& M^{*}(A)=2 \int_{0}^{1} \gamma(a+(1-\gamma) \beta) d \gamma=a+\frac{\beta}{3} .
\end{aligned}
$$


Therefore, the interval-valued possibilistic mean value and crisp possibilistic mean value of $A$ can be written as

$$
\begin{gathered}
M(A)=\left[a-\frac{\alpha}{3}, a+\frac{\beta}{3}\right], \\
\bar{M}(A)=a+\frac{\beta-\alpha}{6} .
\end{gathered}
$$

Finally, the weighted possibilistic mean value of fuzzy number $A$ is computed as

$$
\bar{M}(A ; t)=a+\frac{t \beta-(1-t) \alpha}{3}, \quad t \in[0,1] .
$$

\section{Partner Selection Problem Description and Notations}

Let us consider a core enterprise getting a bid for a large project consisting of several subprojects. The core enterprise cannot complete the whole project only by its own ability. Therefore, the core enterprise has to select partners and form a VE to complete the project. The partner selection problem of a VE is described as follows: $X=\left\{X_{i} \mid i=\right.$ $1, \ldots, n\}$ represents a finite set of possible candidates, $B=$ $\left\{B_{j} \mid j=1, \ldots, m\right\}$ represents a finite set of attributes according to the desirability judgment on a candidate.

Assuming the candidates' information is imprecise and fuzzy, the decision maker DM\# $\mathrm{D}(\kappa=1,2, \ldots, K)$ utilizes a triangular fuzzy number $\tilde{a}_{i j}^{\kappa}$ with center $a_{i j}^{\kappa}$, left-width $\alpha_{i j}^{\kappa}>$ 0 , and right-width $\beta_{i j}^{\kappa}>0$; that is, $\widetilde{a}_{i j}^{\kappa}=\left(a_{i j}^{\kappa}-\alpha_{i j}{ }^{\kappa}, a_{i j}{ }^{\kappa}, a_{i j}{ }^{\kappa}+\right.$ $\left.\beta_{i j}^{\kappa}\right)$ to evaluate candidate $X_{i}$ with respect to attribute $B_{j}$. Let $\widetilde{A}^{\kappa}=\left[\widetilde{a}_{i j}^{\kappa}\right]_{n \times m}$ be the decision matrix in the form of triangular fuzzy numbers. Then the group MADM problem with triangular fuzzy numbers can be expressed in matrix format as follows:

$$
\widetilde{A}^{\kappa}=\left[\begin{array}{cccc}
\tilde{a}_{11}^{\kappa} & \tilde{a}_{12}^{\kappa} & \ldots & \tilde{a}_{1 m}^{\kappa} \\
\tilde{a}_{21}^{\kappa} & \tilde{a}_{22}^{\kappa} & \ldots & \tilde{a}_{2 m}^{\kappa} \\
\vdots & \vdots & \ddots & \vdots \\
\tilde{a}_{n 1}^{\kappa} & \tilde{a}_{n 2}^{\kappa} & \ldots & \tilde{a}_{n m}^{\kappa}
\end{array}\right] .
$$

Each decision maker DM\# $\boldsymbol{D}(\kappa=1,2, \ldots, K)$ will elicit weights for attribute $B_{j}$ as $\omega_{j}^{\kappa}$, where $j=1,2, \ldots, m . \omega_{j}^{\kappa}(j=$ $1,2, \ldots, m)$ belongs to $[0,1]$ and sums to one [18]; that is,

$$
0 \leq \omega_{j}^{\kappa} \leq 1, \quad j=1,2, \ldots, m, \quad \sum_{j=1}^{m} \omega_{j}^{\kappa}=1 .
$$

The values of different attributes have different dimensions. Thus, the fuzzy decision matrix $\widetilde{A}^{\kappa}=\left[\widetilde{a}_{i j}^{\kappa}\right]_{n \times m}(\kappa=1,2$, $\ldots, K)$ should be standardized with matrix $\widetilde{A}^{\prime \kappa}$ in order to reduce disturbance in the final results. Let $\widetilde{A}^{\prime \kappa}=\left[\widetilde{a}_{i j}^{\prime \kappa}\right]_{n \times m}$ be the standardized decision matrix in the form of triangular fuzzy numbers, where $\tilde{a}_{i j}^{\prime \kappa}=\left(a_{i j}^{\prime \kappa}-\alpha_{i j}^{\prime \kappa}, a_{i j}^{\prime \kappa}, a_{i j}^{\prime \kappa}+\beta_{i j}^{\prime \kappa}\right)$.

In general, there are two attribute categories for candidates: benefit type and cost type. The higher the benefit type value is, the better it will be. It is opposite for the cost type. We describe the normalized formula for triangular fuzzy numbers of the benefit type as follows:

$$
\begin{aligned}
\left(a_{i j}^{\prime \kappa}-\alpha_{i j}^{\prime \kappa}\right) & =\frac{\left(a_{i j}^{\kappa}-\alpha_{i j}^{\kappa}\right)}{\sqrt{\sum_{i=1}^{n}\left(a_{i j}^{\kappa}+\beta_{i j}^{\kappa}\right)^{2}}}, \\
a_{i j}^{\prime \kappa} & =\frac{a_{i j}^{\kappa}}{\sqrt{\sum_{i=1}^{n}\left(a_{i j}^{\kappa}\right)^{2}}}, \\
\left(a_{i j}^{\prime \kappa}+\beta_{i j}^{\prime \kappa}\right) & =\frac{\left(a_{i j}^{\kappa}+\beta_{i j}^{\kappa}\right)}{\sqrt{\sum_{i=1}^{n}\left(a_{i j}^{\kappa}-\alpha_{i j}^{\kappa}\right)^{2}}} .
\end{aligned}
$$

Similarly, the formula for the triangular fuzzy number of the cost type is described as follows:

$$
\begin{aligned}
\left(a_{i j}^{\prime \kappa}-\alpha_{i j}^{\prime \kappa}\right) & =\frac{1 /\left(a_{i j}^{\kappa}+\beta_{i j}^{\kappa}\right)}{\sqrt{\sum_{i=1}^{n}\left(1 /\left(a_{i j}^{\kappa}-\alpha_{i j}^{\kappa}\right)\right)^{2}}}, \\
a_{i j}^{\prime \kappa} & =\frac{1 / a_{i j}^{\kappa}}{\sqrt{\sum_{i=1}^{n}\left(1 / a_{i j}^{\kappa}\right)^{2}}}, \\
\left(a_{i j}^{\prime \kappa}+\beta_{i j}^{\prime \kappa}\right) & =\frac{1 /\left(a_{i j}^{\kappa}-\alpha_{i j}^{\kappa}\right)}{\sqrt{\sum_{i=1}^{n}\left(1 /\left(a_{i j}^{\kappa}+\beta_{i j}^{\kappa}\right)\right)^{2}}} .
\end{aligned}
$$

Now the triangular fuzzy number $\tilde{a}_{i j}^{\prime \kappa}=\left(a_{i j}^{\prime \kappa}-\alpha_{i j}^{\prime \kappa}, a_{i j}^{\prime \kappa}\right.$, $\left.a_{i j}^{\prime \kappa}+\beta_{i j}^{\prime \kappa}\right)$ is the normalized form of triangular fuzzy number $\widetilde{a}_{i j}^{\kappa}=\left(a_{i j}^{\kappa}-\alpha_{i j}^{\kappa}, a_{i j}^{\kappa}, a_{i j}^{\kappa}+\beta_{i j}^{\kappa}\right)$.

According to formula (5), we can get the weighted possibilistic mean value of triangular fuzzy number $\widetilde{a}_{i j}^{\prime \kappa}=\left(a_{i j}^{\prime \kappa}-\right.$ $\left.\alpha_{i j}^{\prime \kappa}, a_{i j}^{\prime \kappa}, a_{i j}^{\prime \kappa}+\beta_{i j}^{\prime \kappa}\right)$ as follows:

$$
\bar{M}\left(\tilde{a}_{i j}^{\prime \kappa} ; t^{\kappa}\right)=a_{i j}^{\prime \kappa}+\frac{t^{\kappa} \beta_{i j}^{\prime \kappa}-\left(1-t^{\kappa}\right) \alpha_{i j}^{\prime \kappa}}{3}, \quad t^{\kappa} \in[0,1],
$$

where $t^{\kappa}$ reflects the decision maker DM\# $\kappa$ 's risk preference coefficient; a larger $t^{\kappa}$ implies more risk-prone decision maker, whereas a smaller $t^{\kappa}$ indicates a more risk-averse decision maker. Specially, $t^{\kappa}=0, t^{\kappa}=0.5$, and $t^{\kappa}=1$ represent that the decision maker is extremely risk averse, risk neutral, and extremely risk prone, respectively.

Let $\bar{M}\left(\widetilde{A}^{\prime \kappa} ; t^{\kappa}\right)=\left[\bar{M}\left(\widetilde{a}_{i j}^{\prime \kappa} ; t^{\kappa}\right)\right]_{n \times m}, t^{\kappa} \in[0,1]$, be the decision matrix with the weighted possibilistic mean value of triangular fuzzy number, where $\bar{M}\left(\tilde{a}_{i j}^{\prime \kappa} ; t^{\kappa}\right)=a_{i j}^{\prime \kappa}+\left(t^{\kappa} \beta_{i j}^{\prime \kappa}-\right.$ $\left.\left(1-t^{\kappa}\right) \alpha_{i j}^{\prime \kappa}\right) / 3$. 


\section{An Extended TOPSIS Approach with Weighted Possibilistic Mean Values for Partner Selection}

Yoon [27] developed a TOPSIS for multiattribute decision making. Now, TOPSIS is widely applied across different areas among numerous MADM methods and has received interest from both researchers and practitioners [28]. Hwang and Yoon [29] originally proposed TOPSIS to select the best candidate with a finite number of criteria. Kuo et al. [30] applied fuzzy SAW and fuzzy TOPSIS to select the location of an international distribution center in Asia Pacific. Liao and Kao [24] used fuzzy TOPSIS to select supplier in supply chain management. Boran et al. [31] employed an intuitionistic fuzzy TOPSIS approach to select a sales manager. Behzadian et al. [28] conducted a state-of-the-art literature review on TOPSIS research. Tian et al. [32] developed fuzzy TOPSIS model via chi-square test for information source selection. In this paper, we proposed a new extended TOPSIS method with weighted possibilistic mean values to solve partner selection problem of a VE.

Based on the TOPSIS concept, the chosen candidate should have the shortest distance from the positive ideal partner solution (PIPS) and the farthest distance from the negative ideal partner solution (NIPS). Hence, we identify PIPS $\bar{M}\left(\widetilde{A}^{\prime \kappa} ; t^{\kappa}\right)^{+}$and NIPS $\bar{M}\left(\widetilde{A}^{\prime \kappa} ; t^{\kappa}\right)^{-}$for each decision maker as follows:

$$
\begin{aligned}
& \bar{M}\left(\widetilde{A}^{\prime \kappa} ; t^{\kappa}\right)^{+} \\
& =\left(\bar{M}\left(\tilde{a}_{1}^{\prime \kappa} ; t^{\kappa}\right)^{+}, \bar{M}\left(\tilde{a}_{2}^{\prime \kappa} ; t^{\kappa}\right)^{+}, \bar{M}\left(\tilde{a}_{3}^{\prime \kappa} ; t^{\kappa}\right)^{+}, \ldots, \bar{M}\left(\tilde{a}_{m}^{\prime \kappa} ; t^{\kappa}\right)^{+}\right), \\
& \bar{M}\left(\widetilde{A}^{\kappa} ; t^{\kappa}\right)^{-} \\
& =\left(\bar{M}\left(\tilde{a}_{1}^{\prime \kappa} ; t^{\kappa}\right)^{-}, \bar{M}\left(\tilde{a}_{2}^{\prime \kappa} ; t^{\kappa}\right)^{-}, \bar{M}\left(\tilde{a}_{3}^{\prime \kappa} ; t^{\kappa}\right)^{-}, \ldots, \bar{M}\left(\tilde{a}_{m}^{\prime \kappa} ; t^{\kappa}\right)^{-}\right),
\end{aligned}
$$

where $\bar{M}\left(\tilde{a}_{j}^{\prime \kappa} ; t^{\kappa}\right)^{+}=\max _{i} \bar{M}\left(\tilde{a}_{i j}^{\prime \kappa} ; t^{\kappa}\right), \bar{M}\left(\tilde{a}_{j}^{\prime \kappa} ; t^{\kappa}\right)^{-}=\min _{i} \bar{M}$ $\left(\tilde{a}_{i j}^{\prime \kappa} ; t^{\kappa}\right), i=1,2, \ldots, n$.

By using the $m$-dimensional Euclidean distance, the separation of each candidate from the PIPS $\bar{M}\left(\widetilde{A}^{\kappa} ; t^{\kappa}\right)^{+}$for the decision maker $\mathrm{DM} \# \kappa$ is given as

$$
\begin{aligned}
& d_{i}^{\kappa+}\left(t^{\kappa}\right) \\
& \quad=\sqrt{\sum_{j=1}^{m}\left(\bar{M}\left(\tilde{a}_{i j}^{\prime \kappa} ; t^{\kappa}\right)-\bar{M}\left(\tilde{a}_{i}^{\prime \kappa} ; t^{\kappa}\right)^{+}\right)^{2} \omega_{j}}, \quad i=1,2, \ldots n .
\end{aligned}
$$

Similarly, the separation of each candidate from the NIPS $\bar{M}\left(\widetilde{A}^{\kappa} ; t^{\kappa}\right)^{-}$for the decision maker $\mathrm{DM} \# \kappa$ is given as

$d_{i}^{\kappa-}\left(t^{\kappa}\right)$

$$
=\sqrt{\sum_{j=1}^{m}\left(\bar{M}\left(\tilde{a}_{i j}^{\prime \kappa} ; t^{\kappa}\right)-\bar{M}\left(\tilde{a}_{i}^{\prime \kappa} ; t^{\kappa}\right)^{-}\right)^{2} \omega_{j}}, \quad i=1,2, \ldots n .
$$

A closeness coefficient is defined to determine the ranking of all candidates once $d_{i}^{\kappa+}\left(t^{\kappa}\right)$ and $d_{i}^{\kappa-}\left(t^{\kappa}\right)$ of each candidate $X_{i}$ for decision maker DM\# $\kappa$ is calculated.

The relative closeness of the candidate $X_{i}$ for decision maker $\mathrm{DM} \# \kappa$ is defined as

$$
\begin{array}{r}
\mu_{i}^{\kappa}\left(t^{\kappa}\right)=\frac{d_{i}^{\kappa-}\left(t^{\kappa}\right)}{d_{i}^{\kappa-}\left(t^{\kappa}\right)+d_{i}^{\kappa+}\left(t^{\kappa}\right)}, \quad i=1,2, \ldots n, \\
\kappa=1,2, \ldots, K, t^{\kappa} \in[0,1] .
\end{array}
$$

Obviously, for decision maker $\mathrm{DM} \# \kappa$, the candidate $X_{i}$ is closer to the PIPS and farther from the NIPS as $\mu_{i}{ }^{\kappa}\left(t^{\kappa}\right)$ approaches to 1 . Hence, according to the closeness coefficient, the ranking of all candidates can be determined, and decision maker DM\# $\kappa$ selects the best one among a set of feasible candidates.

In addition, the group separation measure of each candidate is combined through an operation $\otimes$ for all decision makers $\mathrm{DM} \# \kappa(\kappa=1,2, \ldots, K)$. The two group separation measures of the PIPS and NIPS, $\bar{M}\left(\widetilde{A}^{\prime \kappa} ; t^{\kappa}\right)^{+}$and $\bar{M}\left(\widetilde{A}^{\prime \kappa} ; t^{\kappa}\right)^{-}$, respectively, can be computed by the following formulas $[4,33]$ :

$$
\begin{aligned}
D_{i}^{+}= & d_{i}^{1+}\left(t^{1}\right) \oplus d_{i}^{2+}\left(t^{2}\right) \\
& \cdots \oplus d_{i}^{\kappa+}\left(t^{\kappa}\right) \cdots \oplus d_{i}^{\kappa+}\left(t^{\kappa}\right) \text { for candidate } i \\
D_{i}^{-}= & d_{i}^{1-}\left(t^{1}\right) \oplus d_{i}^{2-}\left(t^{2}\right) \\
& \cdots \oplus d_{i}^{\kappa-}\left(t^{\kappa}\right) \cdots \oplus d_{i}^{\kappa-}\left(t^{\kappa}\right) \text { for candidate } i .
\end{aligned}
$$

Here, we take the geometric mean operation to combine all individual separation measures by the following formulas:

$$
\begin{aligned}
& D_{i}^{+}=\left(\prod_{\kappa=1}^{K} d_{i}^{\kappa+}\left(t^{\kappa}\right)\right)^{1 / K} \text { for candidate } i, \\
& D_{i}^{-}=\left(\prod_{\kappa=1}^{K} d_{i}^{\kappa-}\left(t^{\kappa}\right)\right)^{1 / K} \text { for candidate } i .
\end{aligned}
$$

Hence, the relative closeness of the candidate $X_{i}$ is defined as

$$
\mu_{i}=\frac{D_{i}^{-}}{D_{i}^{-}+D_{i}^{+}}, \quad i=1,2, \ldots n
$$

The procedure to find out the best partner with the extended TOPSIS method for group MADM with weighted possibilistic mean values is developed as follows.

Step 1. Make up a group decision-making team $E=\{\mathrm{DM} \# 1$, $\mathrm{DM} \# 2, \ldots, \mathrm{DM} \# \kappa, \ldots, \mathrm{DM} \# K\}$.

Step 2. Generate a set of possible candidates for subproject $X=\left\{X_{i} \mid i=1, \ldots, n\right\}$. 
TABLE 1: The decision matrix with triangular fuzzy numbers and weights of six attributes for each decision maker.

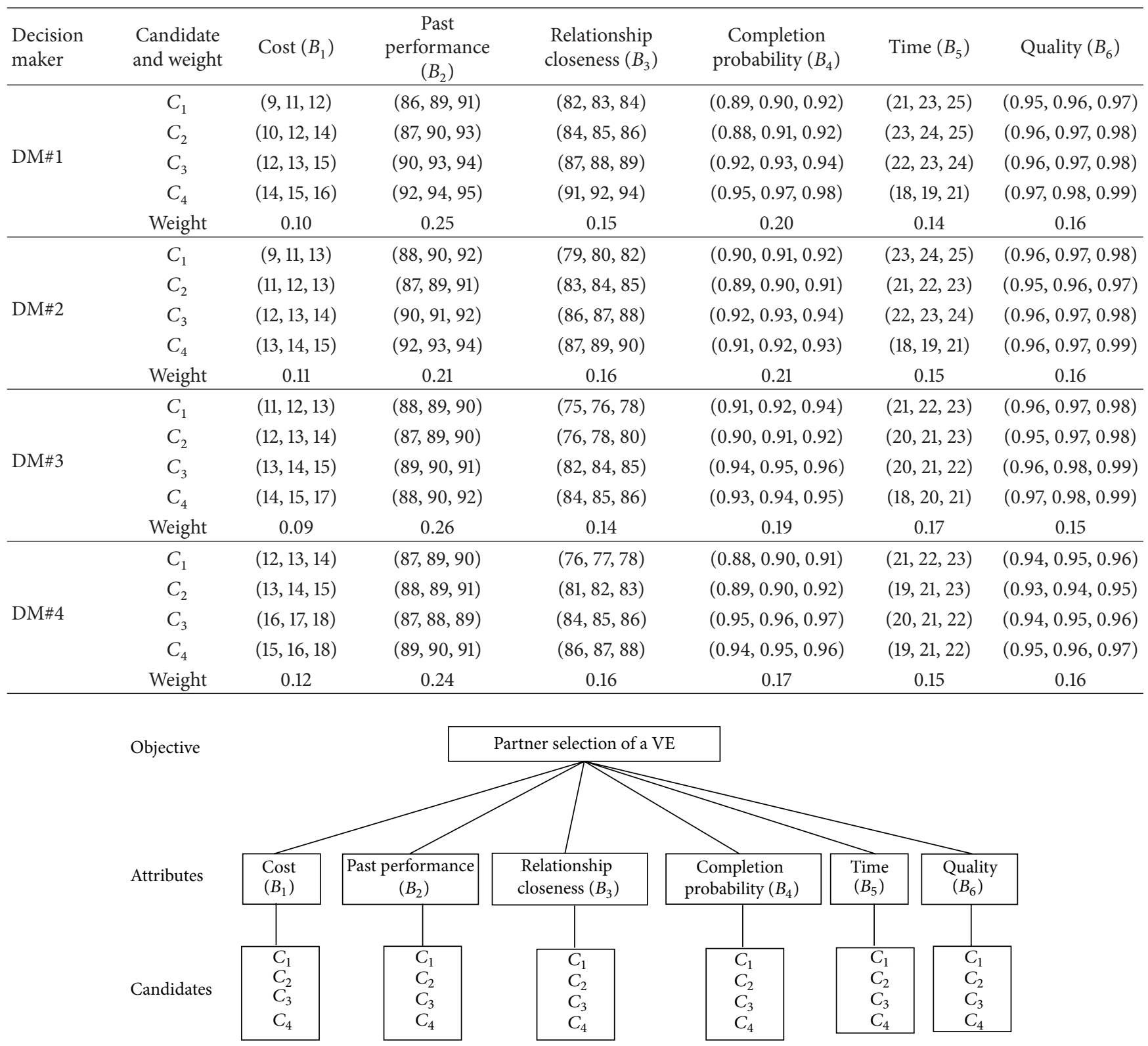

FIGURE 1: Decision hierarchy of the partner selection problem of a VE.

Step 3. Design a set of attributes $B=\left\{B_{j} \mid j=1, \ldots, m\right\}$.

Step 4. Construct the decision matrix $\widetilde{A}^{\kappa}=\left[\widetilde{a}_{i j}^{\kappa}\right]_{n \times m}$ with triangular fuzzy numbers.

Step 5. Each decision maker elicits weights for the attribute $B_{j}$ as $\omega_{j}^{\kappa}$, where $j=1,2, \ldots, m$.

Step 6. Compute normalized ratings. This step tries to transform various attribute dimensions into the nondimensional attributes, which allows comparison between the attributes. Formulas (8) and (9) are used for computing the normalized triangular fuzzy numbers $\tilde{a}_{i j}^{\prime \kappa}=\left(a_{i j}^{\prime \kappa}-\alpha_{i j}^{\prime \kappa}, a_{i j}^{\prime \kappa}, a_{i j}^{\prime \kappa}+\beta_{i j}^{\prime \kappa}\right)$.
Step 7. Calculate weighted possibilistic mean value of triangular fuzzy number $\widetilde{a}_{i j}^{\prime \kappa}=\left(a_{i j}^{\prime \kappa}-\alpha_{i j}^{\prime \kappa}, a_{i j}^{\prime \kappa}, a_{i j}^{\prime \kappa}+\beta_{i j}^{\prime \kappa}\right)$ by using formula (10).

Step 8. Identify PIPS and NIPS by using formula (11).

Step 9. Compute separation measure between candidate and PIPS for each decision maker by using formula (12).

Step 10. Calculate separation measure between candidate and NIPS for each decision maker by using formula (13).

Step 11. Calculate the closeness coefficient of candidate $X_{i}$ for the decision maker DM\# $\kappa$ by using formula (14). 


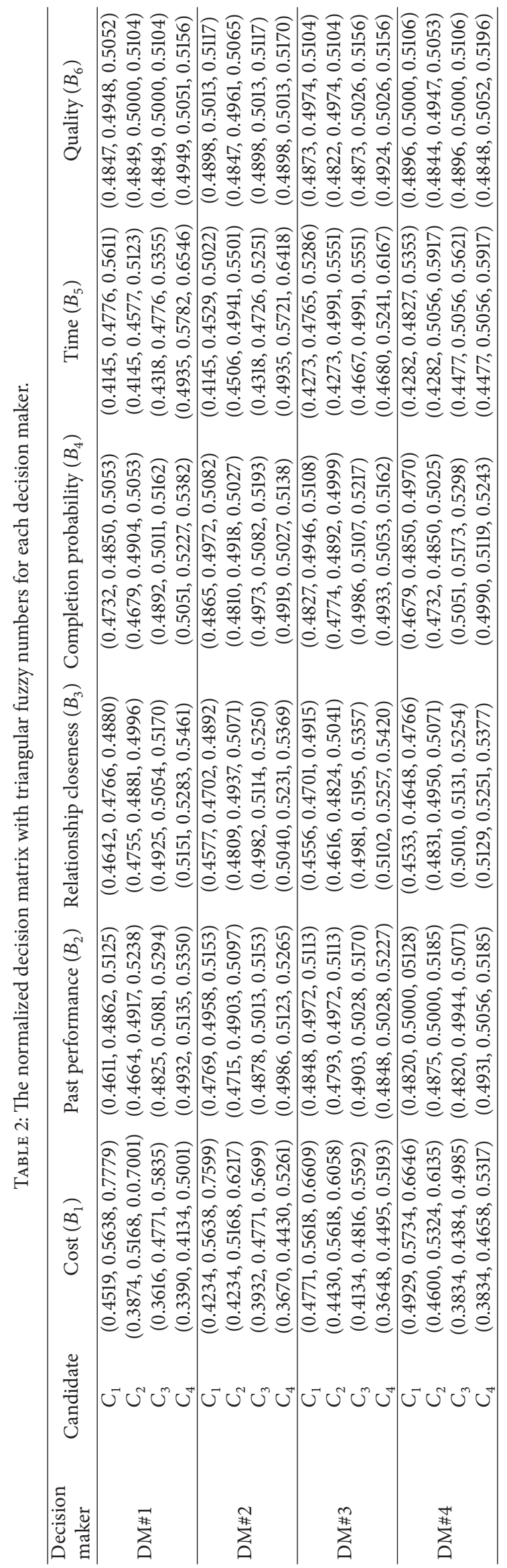


TABLE 3: The normalized decision matrix with weighted possibilistic mean values for each decision maker.

\begin{tabular}{|c|c|c|c|c|c|c|c|c|}
\hline $\begin{array}{l}\text { Decision } \\
\text { maker }\end{array}$ & $t^{\kappa}$ & Candidate & Cost $\left(B_{1}\right)$ & $\begin{array}{c}\text { Past } \\
\text { performance } \\
\left(B_{2}\right)\end{array}$ & $\begin{array}{c}\text { Relationship } \\
\text { closeness }\left(B_{3}\right)\end{array}$ & $\begin{array}{c}\text { Completion } \\
\text { probability }\left(B_{4}\right)\end{array}$ & Time $\left(B_{5}\right)$ & Quality $\left(B_{6}\right)$ \\
\hline \multirow{20}{*}{ DM\#1 } & \multirow{4}{*}{$t^{1}=0$} & $C_{1}$ & 0.5265 & 0.4778 & 0.4725 & 0.4811 & 0.4566 & 0.4914 \\
\hline & & $C_{2}$ & 0.4737 & 0.4833 & 0.4839 & 0.4829 & 0.4433 & 0.4966 \\
\hline & & $C_{3}$ & 0.4386 & 0.4995 & 0.5011 & 0.4972 & 0.4623 & 0.4966 \\
\hline & & $\mathrm{C}_{4}$ & 0.3886 & 0.5068 & 0.5239 & 0.5168 & 0.5499 & 0.5017 \\
\hline & \multirow{4}{*}{$t^{1}=0.3$} & $C_{1}$ & 0.5591 & 0.4830 & 0.4749 & 0.4843 & 0.4712 & 0.4935 \\
\hline & & $C_{2}$ & 0.5049 & 0.4890 & 0.4863 & 0.4866 & 0.4531 & 0.4986 \\
\hline & & $C_{3}$ & 0.4607 & 0.5042 & 0.5035 & 0.4999 & 0.4727 & 0.4986 \\
\hline & & $C_{4}$ & 0.4047 & 0.5109 & 0.5270 & 0.5202 & 0.5660 & 0.5038 \\
\hline & \multirow{4}{*}{$t^{1}=0.5$} & $C_{1}$ & 0.5808 & 0.4864 & 0.4765 & 0.4864 & 0.4810 & 0.4949 \\
\hline & & $C_{2}$ & 0.5258 & 0.4928 & 0.4879 & 0.4891 & 0.4596 & 0.5000 \\
\hline & & $C_{3}$ & 0.4755 & 0.5074 & 0.5052 & 0.5017 & 0.4796 & 0.5000 \\
\hline & & $C_{4}$ & 0.4155 & 0.5137 & 0.5291 & 0.5224 & 0.5768 & 0.5052 \\
\hline & \multirow{4}{*}{$t^{1}=0.7$} & $C_{1}$ & 0.6026 & 0.4898 & 0.4780 & 0.4885 & 0.4908 & 0.4962 \\
\hline & & $C_{2}$ & 0.5466 & 0.4966 & 0.4895 & 0.4916 & 0.4661 & 0.5014 \\
\hline & & $C_{3}$ & 0.4903 & 0.5105 & 0.5068 & 0.5035 & 0.4866 & 0.5014 \\
\hline & & $C_{4}$ & 0.4262 & 0.5165 & 0.5312 & 0.5246 & 0.5875 & 0.5066 \\
\hline & \multirow{4}{*}{$t^{1}=1.0$} & $C_{1}$ & 0.6352 & 0.4950 & 0.4804 & 0.4917 & 0.5054 & 0.4983 \\
\hline & & $C_{2}$ & 0.5779 & 0.5024 & 0.4920 & 0.4953 & 0.4759 & 0.5035 \\
\hline & & $C_{3}$ & 0.5125 & 0.5152 & 0.5093 & 0.5062 & 0.4969 & 0.5035 \\
\hline & & $\mathrm{C}_{4}$ & 0.4423 & 0.5207 & 0.5343 & 0.5279 & 0.6036 & 0.5086 \\
\hline \multirow{20}{*}{$\mathrm{DM} \# 2$} & \multirow{4}{*}{$t^{2}=0$} & $C_{1}$ & 0.5170 & 0.4895 & 0.4660 & 0.4936 & 0.4401 & 0.4975 \\
\hline & & $C_{2}$ & 0.4857 & 0.4840 & 0.4894 & 0.4882 & 0.4796 & 0.4923 \\
\hline & & $C_{3}$ & 0.4491 & 0.4968 & 0.5070 & 0.5045 & 0.4590 & 0.4975 \\
\hline & & $C_{4}$ & 0.4177 & 0.5078 & 0.5168 & 0.4991 & 0.5459 & 0.4975 \\
\hline & \multirow{4}{*}{$t^{2}=0.3$} & $C_{1}$ & 0.5507 & 0.4933 & 0.4692 & 0.4958 & 0.4489 & 0.4996 \\
\hline & & $C_{2}$ & 0.5055 & 0.4879 & 0.4921 & 0.4904 & 0.4896 & 0.4945 \\
\hline & & $C_{3}$ & 0.4668 & 0.4995 & 0.5097 & 0.5067 & 0.4683 & 0.4996 \\
\hline & & $C_{4}$ & 0.4336 & 0.5105 & 0.5200 & 0.5013 & 0.5607 & 0.5002 \\
\hline & \multirow{4}{*}{$t^{2}=0.5$} & $C_{1}$ & 0.5731 & 0.4959 & 0.4713 & 0.4973 & 0.4548 & 0.5011 \\
\hline & & $C_{2}$ & 0.5187 & 0.4904 & 0.4938 & 0.4918 & 0.4962 & 0.4959 \\
\hline & & $C_{3}$ & 0.4786 & 0.5014 & 0.5114 & 0.5082 & 0.4746 & 0.5011 \\
\hline & & $C_{4}$ & 0.4442 & 0.5124 & 0.5222 & 0.5027 & 0.5706 & 0.5020 \\
\hline & \multirow{3}{*}{$t^{2}=0.7$} & $C_{1}$ & 0.5955 & 0.4985 & 0.4734 & 0.4987 & 0.4606 & 0.5026 \\
\hline & & $C_{2}$ & 0.5320 & 0.4929 & 0.4956 & 0.4933 & 0.5028 & 0.4974 \\
\hline & & $C_{3}$ & 0.4903 & 0.5032 & 0.5132 & 0.5097 & 0.4808 & 0.5026 \\
\hline & \multirow{5}{*}{$t^{2}=1.0$} & $C_{4}$ & 0.4548 & 0.5143 & 0.5244 & 0.5042 & 0.5805 & 0.5038 \\
\hline & & $C_{1}$ & 0.6292 & 0.5023 & 0.4765 & 0.5009 & 0.4694 & 0.5048 \\
\hline & & $C_{2}$ & 0.5518 & 0.4968 & 0.4982 & 0.4954 & 0.5128 & 0.4996 \\
\hline & & $C_{3}$ & 0.5080 & 0.5060 & 0.5159 & 0.5119 & 0.4901 & 0.5048 \\
\hline & & $C_{4}$ & 0.4707 & 0.5170 & 0.5277 & 0.5064 & 0.5953 & 0.5065 \\
\hline
\end{tabular}


TABle 3: Continued.

\begin{tabular}{|c|c|c|c|c|c|c|c|c|}
\hline $\begin{array}{l}\text { Decision } \\
\text { maker }\end{array}$ & $t^{\kappa}$ & Candidate & $\operatorname{Cost}\left(B_{1}\right)$ & $\begin{array}{c}\text { Past } \\
\text { performance } \\
\left(B_{2}\right)\end{array}$ & $\begin{array}{l}\text { Relationship } \\
\text { closeness }\left(B_{3}\right)\end{array}$ & $\begin{array}{c}\text { Completion } \\
\text { probability }\left(B_{4}\right)\end{array}$ & Time $\left(B_{5}\right)$ & Quality $\left(B_{6}\right)$ \\
\hline \multirow{20}{*}{$\mathrm{DM} \# 3$} & \multirow{4}{*}{$t^{3}=0$} & $C_{1}$ & 0.5336 & 0.4931 & 0.4652 & 0.4906 & 0.4601 & 0.4941 \\
\hline & & $C_{2}$ & 0.4934 & 0.4912 & 0.4755 & 0.4852 & 0.4752 & 0.4924 \\
\hline & & $C_{3}$ & 0.4589 & 0.4986 & 0.5124 & 0.5067 & 0.4817 & 0.4975 \\
\hline & & $C_{4}$ & 0.4212 & 0.4968 & 0.5206 & 0.5013 & 0.5054 & 0.4992 \\
\hline & \multirow{4}{*}{$t^{3}=0.3$} & $C_{1}$ & 0.5520 & 0.4957 & 0.4688 & 0.4934 & 0.4702 & 0.4964 \\
\hline & & $C_{2}$ & 0.5097 & 0.4944 & 0.4797 & 0.4875 & 0.4880 & 0.4952 \\
\hline & & $C_{3}$ & 0.4734 & 0.5013 & 0.5161 & 0.5090 & 0.4925 & 0.5003 \\
\hline & & $C_{4}$ & 0.4367 & 0.5006 & 0.5237 & 0.5036 & 0.5203 & 0.5015 \\
\hline & \multirow{4}{*}{$t^{3}=0.5$} & $C_{1}$ & 0.5642 & 0.4975 & 0.4712 & 0.4953 & 0.4770 & 0.4979 \\
\hline & & $C_{2}$ & 0.5205 & 0.4966 & 0.4826 & 0.4890 & 0.4965 & 0.4971 \\
\hline & & $C_{3}$ & 0.4832 & 0.5031 & 0.5186 & 0.5105 & 0.4997 & 0.5022 \\
\hline & & $C_{4}$ & 0.4470 & 0.5031 & 0.5258 & 0.5051 & 0.5302 & 0.5030 \\
\hline & \multirow{4}{*}{$t^{3}=0.7$} & $C_{1}$ & 0.5765 & 0.4993 & 0.4736 & 0.4972 & 0.4837 & 0.4994 \\
\hline & & $C_{2}$ & 0.5314 & 0.4987 & 0.4854 & 0.4905 & 0.5050 & 0.4989 \\
\hline & & $C_{3}$ & 0.4929 & 0.5049 & 0.5212 & 0.5120 & 0.5070 & 0.5041 \\
\hline & & $\mathrm{C}_{4}$ & 0.4573 & 0.5056 & 0.5280 & 0.5067 & 0.5401 & 0.5046 \\
\hline & \multirow{4}{*}{$t^{3}=1.0$} & $C_{1}$ & 0.5949 & 0.5019 & 0.4772 & 0.5000 & 0.4939 & 0.5018 \\
\hline & & $C_{2}$ & 0.5477 & 0.5019 & 0.4897 & 0.4928 & 0.5178 & 0.5018 \\
\hline & & $C_{3}$ & 0.5075 & 0.5075 & 0.5249 & 0.5143 & 0.5178 & 0.5069 \\
\hline & & $C_{4}$ & 0.4727 & 0.5094 & 0.5311 & 0.5089 & 0.5550 & 0.5069 \\
\hline \multirow{20}{*}{$\mathrm{DM} \# 4$} & \multirow{4}{*}{$t^{4}=0$} & $C_{1}$ & 0.5465 & 0.4940 & 0.4609 & 0.4793 & 0.4645 & 0.4965 \\
\hline & & $C_{2}$ & 0.5083 & 0.4958 & 0.4910 & 0.4810 & 0.4798 & 0.4913 \\
\hline & & $C_{3}$ & 0.4201 & 0.4902 & 0.5090 & 0.5132 & 0.4863 & 0.4965 \\
\hline & & $C_{4}$ & 0.4384 & 0.5014 & 0.5211 & 0.5079 & 0.4863 & 0.5018 \\
\hline & \multirow{4}{*}{$t^{4}=0.3$} & $C_{1}$ & 0.5637 & 0.4971 & 0.4633 & 0.4822 & 0.4752 & 0.4986 \\
\hline & & $C_{2}$ & 0.5236 & 0.4989 & 0.4934 & 0.4840 & 0.4962 & 0.4934 \\
\hline & & $C_{3}$ & 0.4316 & 0.4927 & 0.5115 & 0.5157 & 0.4978 & 0.4986 \\
\hline & & $\mathrm{C}_{4}$ & 0.4532 & 0.5040 & 0.5235 & 0.5103 & 0.5007 & 0.5039 \\
\hline & \multirow{4}{*}{$t^{4}=0.5$} & $C_{1}$ & 0.5752 & 0.4991 & 0.4648 & 0.4841 & 0.4824 & 0.5000 \\
\hline & & $C_{2}$ & 0.5339 & 0.5010 & 0.4950 & 0.4859 & 0.5071 & 0.4948 \\
\hline & & $C_{3}$ & 0.4393 & 0.4944 & 0.5131 & 0.5173 & 0.5054 & 0.5000 \\
\hline & & $C_{4}$ & 0.4631 & 0.5057 & 0.5252 & 0.5120 & 0.5103 & 0.5053 \\
\hline & \multirow{4}{*}{$t^{4}=0.7$} & $C_{1}$ & 0.5866 & 0.5012 & 0.4664 & 0.4861 & 0.4895 & 0.5014 \\
\hline & & $C_{2}$ & 0.5441 & 0.5031 & 0.4966 & 0.4879 & 0.5180 & 0.4962 \\
\hline & & $C_{3}$ & 0.4469 & 0.4961 & 0.5147 & 0.5190 & 0.5130 & 0.5014 \\
\hline & & $\mathrm{C}_{4}$ & 0.4730 & 0.5074 & 0.5268 & 0.5136 & 0.5199 & 0.5067 \\
\hline & \multirow{4}{*}{$t^{4}=1.0$} & $C_{1}$ & 0.6038 & 0.5043 & 0.4687 & 0.4890 & 0.5002 & 0.5035 \\
\hline & & $C_{2}$ & 0.5594 & 0.5062 & 0.4990 & 0.4908 & 0.5343 & 0.4983 \\
\hline & & $C_{3}$ & 0.4585 & 0.4986 & 0.5172 & 0.5214 & 0.5245 & 0.5035 \\
\hline & & $C_{4}$ & 0.4878 & 0.5099 & 0.5293 & 0.5160 & 0.5343 & 0.5088 \\
\hline
\end{tabular}


TABLE 4: PIPS and NIPS for each decision maker.

\begin{tabular}{|c|c|c|c|c|c|c|c|c|}
\hline $\begin{array}{l}\text { Decision } \\
\text { maker }\end{array}$ & $t^{\kappa}$ & PIPS/NIPS & Cost $\left(B_{1}\right)$ & $\begin{array}{c}\text { Past } \\
\text { performance } \\
\left(B_{2}\right)\end{array}$ & $\begin{array}{l}\text { Relationship } \\
\text { closeness }\left(B_{3}\right)\end{array}$ & $\begin{array}{c}\text { Completion } \\
\text { probability }\left(B_{4}\right)\end{array}$ & Time $\left(B_{5}\right)$ & Quality $\left(B_{6}\right)$ \\
\hline \multirow{10}{*}{ DM\#1 } & \multirow{2}{*}{$t^{1}=0$} & PIPS & 0.5265 & 0.5068 & 0.5239 & 0.5168 & 0.5499 & 0.5017 \\
\hline & & NIPS & 0.3886 & 0.4778 & 0.4725 & 0.4811 & 0.4433 & 0.4914 \\
\hline & \multirow{2}{*}{$t^{1}=0.3$} & PIPS & 0.5591 & 0.5109 & 0.5270 & 0.5202 & 0.5660 & 0.5038 \\
\hline & & NIPS & 0.4047 & 0.4830 & 0.4749 & 0.4843 & 0.4531 & 0.4935 \\
\hline & \multirow{2}{*}{$t^{1}=0.5$} & PIPS & 0.5808 & 0.5137 & 0.5291 & 0.5224 & 0.5768 & 0.5052 \\
\hline & & NIPS & 0.4155 & 0.4864 & 0.4765 & 0.4864 & 0.4596 & 0.4949 \\
\hline & \multirow{2}{*}{$t^{1}=0.7$} & PIPS & 0.6026 & 0.5165 & 0.5312 & 0.5246 & 0.5875 & 0.5066 \\
\hline & & NIPS & 0.4262 & 0.4898 & 0.4780 & 0.4885 & 0.4661 & 0.4962 \\
\hline & \multirow{2}{*}{$t^{1}=1.0$} & PIPS & 0.6352 & 0.5207 & 0.5343 & 0.5279 & 0.6036 & 0.5086 \\
\hline & & NIPS & 0.4423 & 0.4950 & 0.4804 & 0.4917 & 0.4759 & 0.4983 \\
\hline \multirow{10}{*}{$\mathrm{DM} \# 2$} & \multirow{2}{*}{$t^{2}=0$} & PIPS & 0.5170 & 0.5078 & 0.5168 & 0.5045 & 0.5459 & 0.4975 \\
\hline & & NIPS & 0.4177 & 0.4840 & 0.4660 & 0.4882 & 0.4401 & 0.4923 \\
\hline & \multirow{2}{*}{$t^{2}=0.3$} & PIPS & 0.5507 & 0.5105 & 0.5200 & 0.5067 & 0.5607 & 0.5002 \\
\hline & & NIPS & 0.4336 & 0.4879 & 0.4692 & 0.4904 & 0.4489 & 0.4945 \\
\hline & \multirow{2}{*}{$t^{2}=0.5$} & PIPS & 0.5731 & 0.5124 & 0.5222 & 0.5082 & 0.5706 & 0.5020 \\
\hline & & NIPS & 0.4442 & 0.4904 & 0.4713 & 0.4918 & 0.4548 & 0.4959 \\
\hline & \multirow{2}{*}{$t^{2}=0.7$} & PIPS & 0.6026 & 0.5165 & 0.5312 & 0.5246 & 0.5875 & 0.5066 \\
\hline & & NIPS & 0.4262 & 0.4898 & 0.4780 & 0.4885 & 0.4661 & 0.4962 \\
\hline & \multirow{2}{*}{$t^{2}=1.0$} & PIPS & 0.6292 & 0.5170 & 0.5277 & 0.5119 & 0.5953 & 0.5065 \\
\hline & & NIPS & 0.4707 & 0.4968 & 0.4765 & 0.4954 & 0.4694 & 0.4996 \\
\hline \multirow{10}{*}{$\mathrm{DM} \# 3$} & \multirow{2}{*}{$t^{3}=0$} & PIPS & 0.5336 & 0.4986 & 0.5206 & 0.5067 & 0.5054 & 0.4992 \\
\hline & & NIPS & 0.4212 & 0.4912 & 0.4652 & 0.4852 & 0.4601 & 0.4924 \\
\hline & \multirow{2}{*}{$t^{3}=0.3$} & PIPS & 0.5520 & 0.5013 & 0.5237 & 0.5090 & 0.5203 & 0.5015 \\
\hline & & NIPS & 0.4367 & 0.4944 & 0.4688 & 0.4875 & 0.4702 & 0.4952 \\
\hline & \multirow{2}{*}{$t^{3}=0.5$} & PIPS & 0.5642 & 0.5031 & 0.5258 & 0.5105 & 0.5302 & 0.5030 \\
\hline & & NIPS & 0.4470 & 0.4966 & 0.4712 & 0.4890 & 0.4770 & 0.4971 \\
\hline & \multirow{2}{*}{$t^{3}=0.7$} & PIPS & 0.5765 & 0.5056 & 0.5280 & 0.5120 & 0.5401 & 0.5046 \\
\hline & & NIPS & 0.4573 & 0.4987 & 0.4736 & 0.4905 & 0.4837 & 0.4989 \\
\hline & \multirow{2}{*}{$t^{3}=1.0$} & PIPS & 0.5949 & 0.5094 & 0.5311 & 0.5143 & 0.5550 & 0.5069 \\
\hline & & NIPS & 0.4727 & 0.5019 & 0.4772 & 0.4928 & 0.4939 & 0.5018 \\
\hline \multirow{10}{*}{$\mathrm{DM} \# 4$} & \multirow{2}{*}{$t^{4}=0$} & PIPS & 0.5465 & 0.5014 & 0.5211 & 0.5132 & 0.4863 & 0.5018 \\
\hline & & NIPS & 0.4201 & 0.4902 & 0.4609 & 0.4793 & 0.4645 & 0.4913 \\
\hline & \multirow{2}{*}{$t^{4}=0.3$} & PIPS & 0.5637 & 0.5040 & 0.5235 & 0.5157 & 0.5007 & 0.5039 \\
\hline & & NIPS & 0.4316 & 0.4927 & 0.4633 & 0.4822 & 0.4752 & 0.4934 \\
\hline & \multirow{2}{*}{$t^{4}=0.5$} & PIPS & 0.5752 & 0.5057 & 0.5252 & 0.5173 & 0.5103 & 0.5053 \\
\hline & & NIPS & 0.4393 & 0.4944 & 0.4648 & 0.4841 & 0.4824 & 0.4948 \\
\hline & \multirow{2}{*}{$t^{4}=0.7$} & PIPS & 0.5866 & 0.5074 & 0.5268 & 0.5190 & 0.5199 & 0.5067 \\
\hline & & NIPS & 0.4469 & 0.4961 & 0.4664 & 0.4861 & 0.4895 & 0.4962 \\
\hline & \multirow{2}{*}{$t^{4}=1.0$} & PIPS & 0.6038 & 0.5099 & 0.5293 & 0.5214 & 0.5343 & 0.5088 \\
\hline & & NIPS & 0.4585 & 0.4986 & 0.4687 & 0.4890 & 0.5002 & 0.4983 \\
\hline
\end{tabular}

Step 12. Rank the preference order of all candidates according to the closeness coefficient and select the best partner for decision maker $\mathrm{DM} \#$.

Step 13. Aggregate the separation measure for the group by using formula (16).
Step 14. Calculate the closeness coefficient of candidate $X_{i}$ for the group by using formula (17).

Step 15. Rank the preference order of all candidates according to the closeness coefficient and select the best one. 
TABLE 5: Separation measure for each decision maker.

\begin{tabular}{|c|c|c|c|c|c|}
\hline Decision maker & $t^{\kappa}$ & $C_{1}$ & $C_{2}$ & $C_{3}$ & $\mathrm{C}_{4}$ \\
\hline \multirow{10}{*}{ DM\#1 } & \multirow{2}{*}{$t^{1}=0$} & $d_{1}^{1+}=0.0458$ & $d_{2}^{1+}=0.0498$ & $d_{3}^{1+}=0.0450$ & $d_{4}^{1+}=0.0436$ \\
\hline & & $d_{1}^{1-}=0.0439$ & $d_{2}^{1-}=0.0275$ & $d_{3}^{1-}=0.0244$ & $d_{4}^{1-}=0.0497$ \\
\hline & \multirow{2}{*}{$t^{1}=0.3$} & $d_{1}^{1+}=0.0462$ & $d_{2}^{1+}=0.0517$ & $d_{3}^{1+}=0.0487$ & $d_{4}^{1+}=0.0488$ \\
\hline & & $d_{1}^{1-}=0.0493$ & $d_{2}^{1-}=0.0322$ & $d_{3}^{1-}=0.0256$ & $d_{4}^{1-}=0.0516$ \\
\hline & \multirow{2}{*}{$t^{1}=0.5$} & $d_{1}^{1+}=0.0465$ & $d_{2}^{1+}=0.0530$ & $d_{3}^{1+}=0.0511$ & $d_{4}^{1+}=0.0523$ \\
\hline & & $d_{1}^{1-}=0.0529$ & $d_{2}^{1-}=0.0354$ & $d_{3}^{1-}=0.0265$ & $d_{4}^{1-}=0.0529$ \\
\hline & \multirow{2}{*}{$t^{1}=0.7$} & $d_{1}^{1+}=0.0468$ & $d_{2}^{1+}=0.0544$ & $d_{3}^{1+}=0.0537$ & $d_{4}^{1+}=0.0558$ \\
\hline & & $d_{1}^{1-}=0.0565$ & $d_{2}^{1-}=0.0386$ & $d_{3}^{1-}=0.0274$ & $d_{4}^{1-}=0.0542$ \\
\hline & \multirow{2}{*}{$t^{1}=1.0$} & $d_{1}^{1+}=0.0472$ & $d_{2}^{1+}=0.0564$ & $d_{3}^{1+}=0.0574$ & $d_{4}^{1+}=0.0610$ \\
\hline & & $d_{1}^{1-}=0.0620$ & $d_{2}^{1-}=0.0433$ & $d_{3}^{1-}=0.0288$ & $d_{4}^{1-}=0.0562$ \\
\hline \multirow{10}{*}{$\mathrm{DM} \# 2$} & \multirow{2}{*}{$t^{2}=0$} & $d_{1}^{2+}=0.0467$ & $d_{2}^{2+}=0.0326$ & $d_{3}^{2+}=0.0410$ & $d_{4}^{2+}=0.0330$ \\
\hline & & $d_{1}^{2-}=0.0332$ & $d_{2}^{2-}=0.0288$ & $d_{3}^{2-}=0.0229$ & $d_{4}^{2-}=0.0473$ \\
\hline & \multirow{2}{*}{$t^{2}=0.3$} & $d_{1}^{2+}=0.0488$ & $d_{2}^{2+}=0.0358$ & $d_{3}^{2+}=0.0458$ & $d_{4}^{2+}=0.0389$ \\
\hline & & $d_{1}^{2-}=0.0391$ & $d_{2}^{2-}=0.0300$ & $d_{3}^{2-}=0.0230$ & $d_{4}^{2-}=0.0493$ \\
\hline & \multirow{2}{*}{$t^{2}=0.5$} & $d_{1}^{2+}=0.0501$ & $d_{2}^{2+}=0.0381$ & $d_{3}^{2+}=0.0491$ & $d_{4}^{2+}=0.0428$ \\
\hline & & $d_{1}^{2-}=0.0430$ & $d_{2}^{2-}=0.0308$ & $d_{3}^{2-}=0.0231$ & $d_{4}^{2-}=0.0506$ \\
\hline & \multirow{2}{*}{$t^{2}=0.7$} & $d_{1}^{2+}=0.0515$ & $d_{2}^{2+}=0.0405$ & $d_{3}^{2+}=0.0525$ & $d_{4}^{2+}=0.0467$ \\
\hline & & $d_{1}^{2-}=0.0469$ & $d_{2}^{2-}=0.0316$ & $d_{3}^{2-}=0.0232$ & $d_{4}^{2-}=0.0520$ \\
\hline & \multirow{2}{*}{$t^{2}=1.0$} & $d_{1}^{2+}=0.0536$ & $d_{2}^{2+}=0.0444$ & $d_{3}^{2+}=0.0577$ & $d_{4}^{2+}=0.0526$ \\
\hline & & $d_{1}^{2-}=0.0527$ & $d_{2}^{2-}=0.0329$ & $d_{3}^{2-}=0.0233$ & $d_{4}^{2-}=0.0540$ \\
\hline \multirow{10}{*}{$\mathrm{DM} \# 3$} & \multirow{2}{*}{$t^{3}=0$} & $d_{1}^{3+}=0.0290$ & $d_{2}^{3+}=0.0263$ & $d_{3}^{3+}=0.0247$ & $d_{4}^{3+}=0.0338$ \\
\hline & & $d_{1}^{3-}=0.0338$ & $d_{2}^{3-}=0.0229$ & $d_{3}^{3-}=0.0250$ & $d_{4}^{3-}=0.0290$ \\
\hline & \multirow{2}{*}{$t^{3}=0.3$} & $d_{1}^{3+}=0.0301$ & $d_{2}^{3+}=0.0267$ & $d_{3}^{3+}=0.0263$ & $d_{4}^{3+}=0.0347$ \\
\hline & & $d_{1}^{3-}=0.0347$ & $d_{2}^{3-}=0.0235$ & $d_{3}^{3-}=0.0250$ & $d_{4}^{3-}=0.0302$ \\
\hline & \multirow{2}{*}{$t^{3}=0.5$} & $d_{1}^{3+}=0.0309$ & $d_{2}^{3+}=0.0270$ & $d_{3}^{3+}=0.0275$ & $d_{4}^{3+}=0.0352$ \\
\hline & & $d_{1}^{3-}=0.0353$ & $d_{2}^{3-}=0.0239$ & $d_{3}^{3-}=0.0250$ & $d_{4}^{3-}=0.0311$ \\
\hline & \multirow{2}{*}{$t^{3}=0.7$} & $d_{1}^{3+}=0.0318$ & $d_{2}^{3+}=0.0274$ & $d_{3}^{3+}=0.0287$ & $d_{4}^{3+}=0.0358$ \\
\hline & & $d_{1}^{3-}=0.0359$ & $d_{2}^{3-}=0.0243$ & $d_{3}^{3-}=0.0250$ & $d_{4}^{3-}=0.0319$ \\
\hline & \multirow{2}{*}{$t^{3}=1.0$} & $d_{1}^{3+}=0.0332$ & $d_{2}^{3+}=0.0280$ & $d_{3}^{3+}=0.0305$ & $d_{4}^{3+}=0.0367$ \\
\hline & & $d_{1}^{3-}=0.0368$ & $d_{2}^{3-}=0.0250$ & $d_{3}^{3-}=0.0250$ & $d_{4}^{3-}=0.0333$ \\
\hline \multirow{10}{*}{$\mathrm{DM} \# 4$} & \multirow{2}{*}{$t^{4}=0$} & $d_{1}^{4+}=0.0294$ & $d_{2}^{4+}=0.0230$ & $d_{3}^{4+}=0.0445$ & $d_{4}^{4+}=0.0375$ \\
\hline & & $d_{1}^{4-}=0.0457$ & $d_{2}^{4-}=0.0352$ & $d_{3}^{4-}=0.0283$ & $d_{4}^{4-}=0.0329$ \\
\hline & \multirow{2}{*}{$t^{4}=0.3$} & $d_{1}^{4+}=0.0298$ & $d_{2}^{4+}=0.0232$ & $d_{3}^{4+}=0.0464$ & $d_{4}^{4+}=0.0383$ \\
\hline & & $d_{1}^{4-}=0.0459$ & $d_{2}^{4-}=0.0352$ & $d_{3}^{4-}=0.0254$ & $d_{4}^{4-}=0.0303$ \\
\hline & \multirow{2}{*}{$t^{4}=0.5$} & $d_{1}^{4+}=0.0300$ & $d_{2}^{4+}=0.0233$ & $d_{3}^{4+}=0.0477$ & $d_{4}^{4+}=0.0389$ \\
\hline & & $d_{1}^{4-}=0.0472$ & $d_{2}^{4-}=0.0364$ & $d_{3}^{4-}=0.0254$ & $d_{4}^{4-}=0.0308$ \\
\hline & \multirow{2}{*}{$t^{4}=0.7$} & $d_{1}^{4+}=0.0304$ & $d_{2}^{4+}=0.0235$ & $d_{3}^{4+}=0.0491$ & $d_{4}^{4+}=0.0394$ \\
\hline & & $d_{1}^{4-}=0.0485$ & $d_{2}^{4-}=0.0376$ & $d_{3}^{4-}=0.0254$ & $d_{4}^{4-}=0.0313$ \\
\hline & \multirow{2}{*}{$t^{4}=1.0$} & $d_{1}^{4+}=0.0309$ & $d_{2}^{4+}=0.0237$ & $d_{3}^{4+}=0.0511$ & $d_{4}^{4+}=0.0402$ \\
\hline & & $d_{1}^{4-}=0.0505$ & $d_{2}^{4-}=0.0395$ & $d_{3}^{4-}=0.0255$ & $d_{4}^{4-}=0.0322$ \\
\hline
\end{tabular}

\section{Numerical Example}

We present a numerical example to illustrate our proposed approach. Four decision makers, namely, DM\#1, DM\#2, $\mathrm{DM} \#$, and DM\#4, from the core enterprise are selected to form the decision-making team. These decision makers are then tasked to select the best partner out of four candidates, namely, $C_{1}, C_{2}, C_{3}$, and $C_{4}$, in forming a new $\mathrm{VE}$. The six attributes of each candidate, including cost $\left(B_{1}\right)$, past performance $\left(B_{2}\right)$, relationship closeness $\left(B_{3}\right)$, completion probability $\left(B_{4}\right)$, time $\left(B_{5}\right)$, and quality $\left(B_{6}\right)$, are considered in the selection process. $B_{1}$ and $B_{5}$ are included in the costtype attributes, whereas $B_{2}, B_{3}, B_{4}$, and $B_{6}$ are included in the benefit-type attributes. Figure 1 shows the hierarchical structure of the partner selection problem, and Table 1 shows the decision matrix that contains the triangular fuzzy numbers and weights of the six attributes of each decision maker.

The selection process is conducted by the procedures outlined in Section 4. We calculate the normalized decision matrix of each decision maker with triangular fuzzy numbers, 
TABLE 6: Closeness coefficient and ranking of each candidate for each decision maker.

\begin{tabular}{|c|c|c|c|c|c|c|c|c|c|c|c|}
\hline \multirow{2}{*}{ Decision maker } & \multirow{2}{*}{ Candidate } & \multicolumn{2}{|c|}{$t^{\kappa}=0$} & \multicolumn{2}{|c|}{$t^{\kappa}=0.3$} & \multicolumn{2}{|c|}{$t^{\kappa}=0.5$} & \multicolumn{2}{|c|}{$t^{\kappa}=0.7$} & \multicolumn{2}{|c|}{$t^{\kappa}=1.0$} \\
\hline & & $\mu_{i}^{\kappa}\left(t^{\kappa}\right)$ & Ranking & $\mu_{i}^{\kappa}\left(t^{\kappa}\right)$ & Ranking & $\mu_{i}^{\kappa}\left(t^{\kappa}\right)$ & Ranking & $\mu_{i}^{\kappa}\left(t^{\kappa}\right)$ & Ranking & $\mu_{i}^{\kappa}\left(t^{\kappa}\right)$ & Ranking \\
\hline \multirow{4}{*}{ DM\#1 } & $C_{1}$ & 0.4892 & 2 & 0.5160 & 1 & 0.5322 & 1 & 0.5472 & 1 & 0.5676 & 1 \\
\hline & $\mathrm{C}_{2}$ & 0.3554 & 3 & 0.3838 & 3 & 0.4002 & 3 & 0.4150 & 3 & 0.4346 & 3 \\
\hline & $C_{3}$ & 0.3520 & 4 & 0.3450 & 4 & 0.3411 & 4 & 0.3378 & 4 & 0.3337 & 4 \\
\hline & $\mathrm{C}_{4}$ & 0.5327 & 1 & 0.5139 & 2 & 0.5030 & 2 & 0.4930 & 2 & 0.4797 & 2 \\
\hline \multirow{4}{*}{$\mathrm{DM} \# 2$} & $C_{1}$ & 0.4154 & 3 & 0.4448 & 3 & 0.4615 & 2 & 0.4765 & 2 & 0.4960 & 2 \\
\hline & $\mathrm{C}_{2}$ & 0.4689 & 2 & 0.4563 & 2 & 0.4474 & 3 & 0.4385 & 3 & 0.4254 & 3 \\
\hline & $C_{3}$ & 0.3586 & 4 & 0.3344 & 4 & 0.3198 & 4 & 0.3063 & 4 & 0.2882 & 4 \\
\hline & $\mathrm{C}_{4}$ & 0.5887 & 1 & 0.5587 & 1 & 0.5416 & 1 & 0.5264 & 1 & 0.5066 & 1 \\
\hline \multirow{4}{*}{ DM\#3 } & $C_{1}$ & 0.5385 & 1 & 0.5354 & 1 & 0.5330 & 1 & 0.5302 & 1 & 0.5257 & 1 \\
\hline & $\mathrm{C}_{2}$ & 0.4647 & 3 & 0.4673 & 3 & 0.4689 & 3 & 0.4701 & 3 & 0.4717 & 3 \\
\hline & $C_{3}$ & 0.5031 & 2 & 0.4865 & 2 & 0.4759 & 2 & 0.4655 & 4 & 0.4506 & 4 \\
\hline & $\mathrm{C}_{4}$ & 0.4620 & 4 & 0.4658 & 4 & 0.4685 & 4 & 0.4714 & 2 & 0.4758 & 2 \\
\hline \multirow{4}{*}{$\mathrm{DM} \# 4$} & $C_{1}$ & 0.6085 & 1 & 0.6065 & 1 & 0.6109 & 1 & 0.6150 & 2 & 0.6204 & 2 \\
\hline & $C_{2}$ & 0.6048 & 2 & 0.6030 & 2 & 0.6095 & 2 & 0.6156 & 1 & 0.6245 & 1 \\
\hline & $C_{3}$ & 0.3890 & 4 & 0.3534 & 4 & 0.3473 & 4 & 0.3413 & 4 & 0.3327 & 4 \\
\hline & $C_{4}$ & 0.4669 & 3 & 0.4412 & 3 & 0.4419 & 3 & 0.4428 & 3 & 0.4447 & 3 \\
\hline
\end{tabular}

TABLE 7: Aggregated separation measure, closeness coefficient, and ranking of candidates.

\begin{tabular}{|c|c|c|c|c|c|c|c|}
\hline Case & Decision maker & $t^{\kappa}$ & Candidate & $\begin{array}{l}\text { Aggregated positive } \\
\text { separation measure }\end{array}$ & $\begin{array}{l}\text { Aggregated negative } \\
\text { separation measure }\end{array}$ & $\mu_{i}$ & Ranking \\
\hline \multirow{4}{*}{ Case no. 1} & $\mathrm{DM} \# 1$ & $t^{1}=0.0$ & $C_{1}$ & $D_{1}^{+}=0.0367$ & $D_{1}^{-}=0.0387$ & 0.5132 & 1 \\
\hline & $\mathrm{DM} \# 2$ & $t^{2}=0.0$ & $\mathrm{C}_{2}$ & $D_{2}^{+}=0.0315$ & $D_{2}^{-}=0.0282$ & 0.4728 & 3 \\
\hline & $\mathrm{DM} \# 3$ & $t^{3}=0.0$ & $\mathrm{C}_{3}$ & $D_{3}^{+}=0.0377$ & $D_{3}^{-}=0.0251$ & 0.3995 & 4 \\
\hline & $\mathrm{DM} \# 4$ & $t^{4}=0.0$ & $\mathrm{C}_{4}$ & $D_{4}^{+}=0.0368$ & $D_{4}^{-}=0.0387$ & 0.5128 & 2 \\
\hline \multirow{4}{*}{ Case no. 2} & $\mathrm{DM} \# 1$ & $t^{1}=0.3$ & $C_{1}$ & $D_{1}^{+}=0.0377$ & $D_{1}^{-}=0.0418$ & 0.5260 & 1 \\
\hline & $\mathrm{DM} \# 2$ & $t^{2}=0.3$ & $C_{2}$ & $D_{2}^{+}=0.0327$ & $D_{2}^{-}=0.0299$ & 0.4774 & 3 \\
\hline & $\mathrm{DM} \# 3$ & $t^{3}=0.3$ & $C_{3}$ & $D_{3}^{+}=0.0406$ & $D_{3}^{-}=0.0247$ & 0.3783 & 4 \\
\hline & $\mathrm{DM} \# 4$ & $t^{4}=0.3$ & $C_{4}$ & $D_{4}^{+}=0.0399$ & $D_{4}^{-}=0.0391$ & 0.4949 & 2 \\
\hline \multirow{4}{*}{ Case no. 3} & $\mathrm{DM} \# 1$ & $t^{1}=0.5$ & $C_{1}$ & $D_{1}^{+}=0.0384$ & $D_{1}^{-}=0.0441$ & 0.5348 & 1 \\
\hline & $\mathrm{DM} \# 2$ & $t^{2}=0.5$ & $\mathrm{C}_{2}$ & $D_{2}^{+}=0.0336$ & $D_{2}^{-}=0.0312$ & 0.4816 & 3 \\
\hline & $\mathrm{DM} \# 3$ & $t^{3}=0.5$ & $C_{3}$ & $D_{3}^{+}=0.0426$ & $D_{3}^{-}=0.0250$ & 0.3693 & 4 \\
\hline & $\mathrm{DM} \# 4$ & $t^{4}=0.5$ & $\mathrm{C}_{4}$ & $D_{4}^{+}=0.0419$ & $D_{4}^{-}=0.0400$ & 0.4887 & 2 \\
\hline \multirow{4}{*}{ Case no. 4} & $\mathrm{DM} \# 1$ & $t^{1}=0.0$ & $C_{1}$ & $D_{1}^{+}=0.0381$ & $D_{1}^{-}=0.0414$ & 0.5210 & 1 \\
\hline & $\mathrm{DM} \# 2$ & $t^{2}=0.3$ & $C_{2}$ & $D_{2}^{+}=0.0326$ & $D_{2}^{-}=0.0293$ & 0.4735 & 3 \\
\hline & $\mathrm{DM} \# 3$ & $t^{3}=0.5$ & $C_{3}$ & $D_{3}^{+}=0.0392$ & $D_{3}^{-}=0.0244$ & 0.3745 & 4 \\
\hline & $\mathrm{DM} \# 4$ & $t^{4}=0.7$ & $C_{4}$ & $D_{4}^{+}=0.0195$ & $D_{4}^{-}=0.0393$ & 0.5007 & 2 \\
\hline \multirow{4}{*}{ Case no. 5} & $\mathrm{DM} \# 1$ & $t^{1}=0.3$ & $C_{1}$ & $D_{1}^{+}=0.0388$ & $D_{1}^{-}=0.0442$ & 0.5326 & 1 \\
\hline & $\mathrm{DM} \# 2$ & $t^{2}=0.5$ & $\mathrm{C}_{2}$ & $D_{2}^{+}=0.0336$ & $D_{2}^{-}=0.0312$ & 0.4815 & 3 \\
\hline & $\mathrm{DM} \# 3$ & $t^{3}=0.7$ & $C_{3}$ & $D_{3}^{+}=0.0433$ & $D_{3}^{-}=0.0248$ & 0.3641 & 4 \\
\hline & $\mathrm{DM} \# 4$ & $t^{4}=1.0$ & $\mathrm{C}_{4}$ & $D_{4}^{+}=0.0417$ & $D_{4}^{-}=0.0405$ & 0.4929 & 2 \\
\hline \multirow{4}{*}{ Case no. 6} & $\mathrm{DM} \# 1$ & $t^{1}=0.7$ & $C_{1}$ & $D_{1}^{+}=0.0390$ & $D_{1}^{-}=0.0463$ & 0.5427 & 1 \\
\hline & $\mathrm{DM} \# 2$ & $t^{2}=0.7$ & $C_{2}$ & $D_{2}^{+}=0.0345$ & $D_{2}^{-}=0.0325$ & 0.4850 & 2 \\
\hline & $\mathrm{DM} \# 3$ & $t^{3}=0.7$ & $C_{3}$ & $D_{3}^{+}=0.0446$ & $D_{3}^{-}=0.0252$ & 0.3609 & 4 \\
\hline & $\mathrm{DM} \# 4$ & $t^{4}=0.7$ & $C_{4}$ & $D_{4}^{+}=0.0438$ & $D_{4}^{-}=0.0410$ & 0.4834 & 3 \\
\hline \multirow{4}{*}{ Case no. 7} & $\mathrm{DM} \# 1$ & $t^{1}=1.0$ & $C_{1}$ & $D_{1}^{+}=0.0401$ & $D_{1}^{-}=0.0496$ & 0.5529 & 1 \\
\hline & $\mathrm{DM} \# 2$ & $t^{2}=1.0$ & $\mathrm{C}_{2}$ & $D_{2}^{+}=0.0359$ & $D_{2}^{-}=0.0344$ & 0.4895 & 2 \\
\hline & $\mathrm{DM} \# 3$ & $t^{3}=1.0$ & $C_{3}$ & $D_{3}^{+}=0.0476$ & $D_{3}^{-}=0.0256$ & 0.3492 & 4 \\
\hline & $\mathrm{DM} \# 4$ & $t^{4}=1.0$ & $\mathrm{C}_{4}$ & $D_{4}^{+}=0.0467$ & $D_{4}^{-}=0.0425$ & 0.4767 & 3 \\
\hline
\end{tabular}




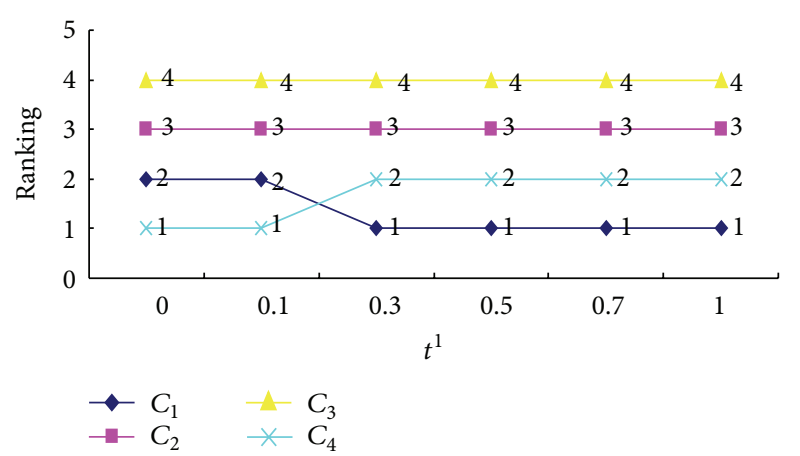

FIGURE 2: The change of ranking of candidates with the change of $t^{1}$ for decision maker DM\#1.

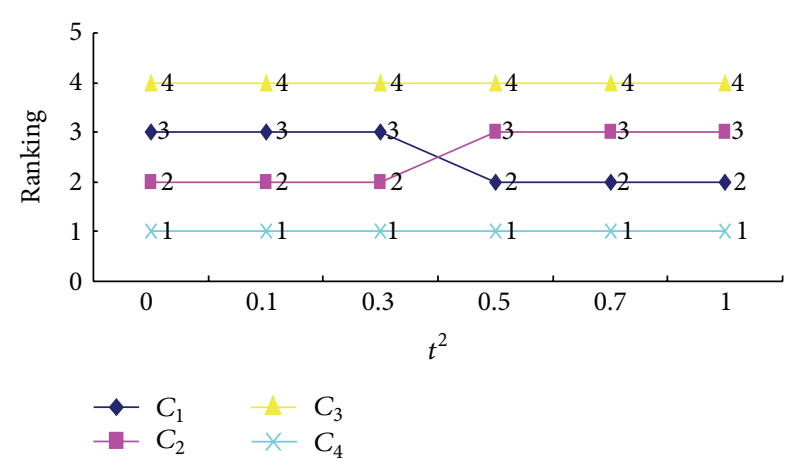

FIGURE 3: The change of ranking of candidates with the change of $t^{2}$ for decision maker DM\#2.

as well as the normalized decision matrix for each decision maker with weighted possibilistic mean values; the results are shown in Tables 2 and 3.

We identify the PIPS and NIPS of each decision maker by using (11); the results are shown in Table 4. The positive and negative separation measures between each candidate for each decision maker are calculated by using (12) and (13), respectively; the results are shown in Table 5.

The closeness coefficient is used to determine the preference order of each candidate for each decision maker; the results are shown in Table 6 and Figures 2, 3, 4, and 5.

For DM\#1, the candidates are ranked as $C_{4}>C_{1}>C_{2}>$ $C_{3}$ when $t^{1}=0(\succ=$ superior to $)$ but are ranked as $C_{1}>$ $C_{4}>C_{2}>C_{3}$ when $t^{1}=0.3,0.5,0.7,1.0$. For DM\#2, the candidates are ranked as $C_{4}>C_{2}>C_{1}>C_{3}$ when $t^{2}=$ 0 or 0.3 but are ranked as $C_{4}>C_{1}>C_{2}>C_{3}$ when $t^{2}=$ $0.5,0.7,1.0$.Therefore, $C_{1}$ is identified as the best candidate for $\mathrm{DM} \# 3$, and $C_{1}$ or $C_{2}$ may be chosen as the partner for DM\#4.

Decision makers may experience problems in reaching a consensus during a group decision-making process. Therefore, these decision makers must combine their assessments to form a highly reasonable evaluation. We obtain the aggregated group separation measures of all candidates by using (16); the results are shown in Table 7. The aggregated closed closeness coefficients and ranking of all candidates are calculated by using (17); the results are shown in Table 7 and Figure 6. $C_{1}$ is identified as the best candidate in all four cases.

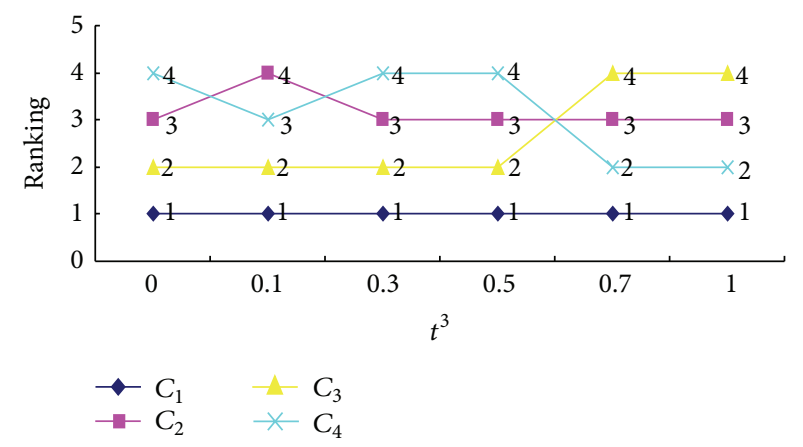

FIGURE 4: The change of ranking of candidates with the change of $t^{3}$ for decision maker DM\#3.

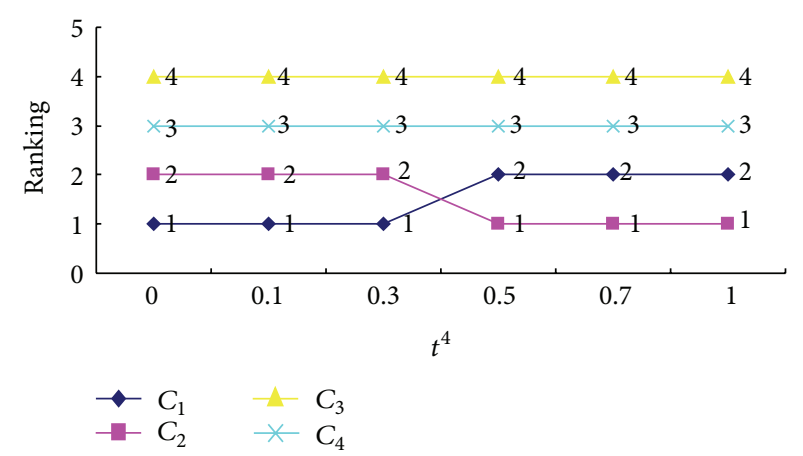

FIGURE 5: The change of ranking of candidates with the change of $t^{4}$ for decision maker DM\#4.

\section{Discussions and Conclusions}

We have proposed an extended TOPSIS for partner selection in a VE. Different from traditional methods, our proposed method considers the varying risk preferences of decision makers. We also use weighted possibilistic mean values to manage candidate information formed by triangular fuzzy numbers.

We perform sensitivity analyses by using a wide range of risk preference coefficient $t$ of decision makers to determine the effects of risk preferences on candidate rank. The value of $t$ may range between zero and one, which covers three categories, namely, extremely risk averse, risk neutral, and extremely risk prone. Our numerical examples show that the ranking of candidates changes with the shifting risk preferences of decision makers. For example, for the first decision maker $(\mathrm{DM \#})$, the fourth candidate $\left(C_{4}\right)$ is selected as the best partner when DM\#1 is extremely risk averse $\left(t^{1}=0\right)$ and the first candidate $\left(C_{1}\right)$ is selected as the best partner when $\mathrm{DM} \# 1$ is less risk averse, risk neutral, or extremely risk prone $\left(t^{1}=0.3,0.5,0.7\right.$, or 1$)$. Other decision makers exhibit the same behavior in their selections. These observations confirm that the risk preferences of decision makers must be considered to produce results that closely reflect real-life practices. Our proposed approach can guide the core enterprise of a VE in selecting a suitable partner, particularly during situations when decision makers exhibit varying risk preferences. 


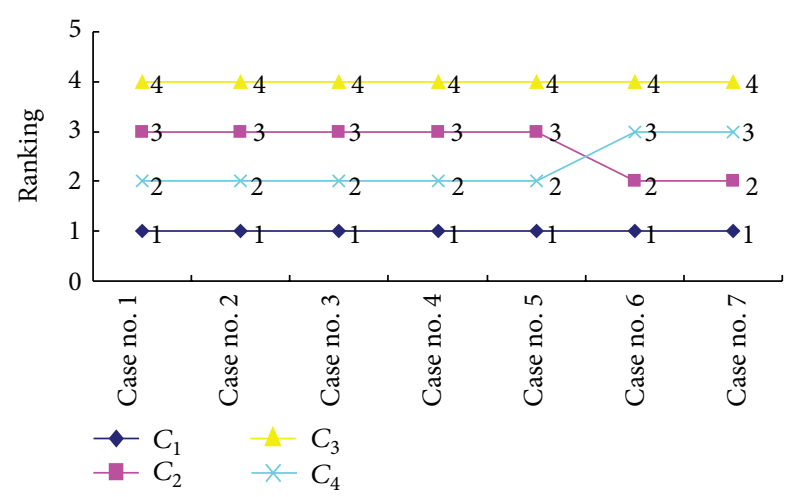

FIGURE 6: The change of ranking of candidates with different risk preference combination cases for the group decision making.

Our extended TOPSIS method may also be applied for other purposes, such as for performance evaluation and for the selection of green suppliers, information systems, facility locations, construction technique alternatives, and human resource arrangements. Future research may investigate the group MADM model with the weighted possibilistic mean values of trapezoidal fuzzy numbers and include the weighted possibilistic variance of fuzzy numbers in the MADM model.

\section{Acknowledgments}

This research is supported by Natural Science Foundation of China (71001041, 71172075, 71371006, and 71090403), Program for New Century Excellent Talents in University (NCET-13-0219), Research Fund for the Doctoral Program of Higher Education of China (20130172110029), and the Fundamental Research Funds for the Central Universities, SCUT, (2013ZZ0093, x2gsD2133310).

\section{References}

[1] W. H. Ip, M. Huang, K. L. Yung, and D. Wang, "Genetic algorithm solution for a risk-based partner selection problem in a virtual enterprise," Computers and Operations Research, vol. 30, no. 2, pp. 213-231, 2003.

[2] N. Wu and P. Su, "Selection of partners in virtual enterprise paradigm," Robotics and Computer-Integrated Manufacturing, vol. 21, no. 2, pp. 119-131, 2005.

[3] J. A. Crispim and J. P. de Sousa, "Partner selection in virtual enterprises: a multi-criteria decision support approach," International Journal of Production Research, vol. 47, no. 17, pp. 47914812, 2009.

[4] F. Ye and Y.-N. Li, "Group multi-attribute decision model to partner selection in the formation of virtual enterprise under incomplete information," Expert Systems with Applications, vol. 36, no. 5, pp. 9350-9357, 2009.

[5] F. Ye, "An extended TOPSIS method with interval-valued intuitionistic fuzzy numbers for virtual enterprise partner selection," Expert Systems with Applications, vol. 37, no. 10, pp. 7050-7055, 2010.

[6] P. Liu, B. Raahemi, and M. Benyoucef, "Knowledge sharing in dynamic virtual enterprises: a socio-technological perspective," Knowledge-Based Systems, vol. 24, no. 3, pp. 427-443, 2011.
[7] S. Talluri and R. C. Baker, "Quantitative framework for designing efficient business process alliances," in Proceedings of the IEEE International Engineering Management Conference, pp. 656-661, Piscataway, NJ, USA, August 1996.

[8] N. Wu, N. Mao, and Y. Qian, "Approach to partner selection in agile manufacturing," Journal of Intelligent Manufacturing, vol. 10 , no. 6, pp. 519-529, 1999.

[9] T. Jarimo and U. Pulkkinen, "A multi-criteria mathematical programming model for agile virtual organization creation," in Network-Centric Collaboration and Supporting Frameworks, L. M. Camarinha-Matos, H. Afsarmanesh, and M. Ollus, Eds., IFIP International Federation for Information Processing, Springer, Boston, Mass, USA, 2005.

[10] Q. Zhao, X. Zhang, and R. Xiao, "Particle swarm optimization algorithm for partner selection in virtual enterprise," Progress in Natural Science, vol. 18, no. 11, pp. 1445-1452, 2008.

[11] W. L. Ng, "An efficient and simple model for multiple criteria supplier selection problem," European Journal of Operational Research, vol. 186, no. 3, pp. 1059-1067, 2008.

[12] D. Y. Sha and Z. H. Che, "Virtual integration with a multicriteria partner selection model for the multi-echelon manufacturing system," International Journal of Advanced Manufacturing Technology, vol. 25, no. 7-8, pp. 793-802, 2005.

[13] B. Sari, T. Sen, and S. E. Kilic, "Ahp model for the selection of partner companies in virtual enterprises," International Journal of Advanced Manufacturing Technology, vol. 38, no. 3-4, pp. 367376, 2008.

[14] D. Wu, "Supplier selection: a hybrid model using DEA, decision tree and neural network," Expert Systems with Applications, vol. 36, no. 5, pp. 9105-9112, 2009.

[15] J. J. H. Liou, "Developing an integrated model for the selection of strategic alliance partners in the airline industry," KnowledgeBased Systems, vol. 28, pp. 59-67, 2012.

[16] L. Mikhailov, "Fuzzy analytical approach to partnership selection in formation of virtual enterprises," Omega, vol. 30, no. 5, pp. 393-401, 2002.

[17] C. Kahraman, U. Cebeci, and Z. lukan, "Multi-criteria supplier selection using fuzzy AHP," Logistics Information Management, vol. 16, pp. 382-394, 2003.

[18] J. Wang and H.-Y. Lin, "A fuzzy hybrid decision-aid model for selecting partners in the design chain," International Journal of Production Research, vol. 44, no. 10, pp. 2047-2069, 2006.

[19] A. Golec and E. Kahya, "A fuzzy model for competency-based employee evaluation and selection," Computers and Industrial Engineering, vol. 52, no. 1, pp. 143-161, 2007.

[20] A. F. Guneri, A. Yucel, and G. Ayyildiz, "An integrated fuzzylp approach for a supplier selection problem in supply chain management," Expert Systems with Applications, vol. 36, no. 5, pp. 9223-9228, 2009.

[21] J. A. Crispim and J. P. de Sousa, "Partner selection in virtual enterprises," International Journal of Production Research, vol. 48, no. 3, pp. 683-707, 2010.

[22] M.-L. Tseng, "Green supply chain management with linguistic preferences and incomplete information," Applied Soft Computing, vol. 11, no. 8, pp. 4894-4903, 2011.

[23] K. Shaw, R. Shankar, S. S. Yadav, and L. S. Thakur, "Supplier selection using fuzzy AHP and fuzzy multi-objective linear programming for developing low carbon supply chain," Expert Systems with Applications, vol. 39, no. 9, pp. 8182-8192, 2012. 
[24] C.-N. Liao and H.-P. Kao, "An integrated fuzzy TOPSIS and MCGP approach to supplier selection in supply chain management," Expert Systems with Applications, vol. 38, no. 9, pp. 10803-10811, 2011.

[25] C. Carlsson and R. Fullér, "On possibilistic mean value and variance of fuzzy numbers," Fuzzy Sets and Systems, vol. 122, no. 2, pp. 315-326, 2001.

[26] W.-G. Zhang, X.-L. Zhang, and W.-L. Xiao, "Portfolio selection under possibilistic mean-variance utility and a SMO algorithm," European Journal of Operational Research, vol. 197, no. 2, pp. 693-700, 2009.

[27] K. Yoon, System selection by multiple attribute decision making [Ph.D. dissertation], Kansas State University, Manhattan, Kan, USA, 1980

[28] M. Behzadian, S. K. Otaghsara, M. Yazdani, and J. Ignatius, "A state-of-the-art survey of TOPSIS applications," Expert Systems with Applications, vol. 39, pp. 13051-13069, 2012.

[29] C. L. Hwang and K. Yoon, Multiple Attribute Decision Making: Methods and Applications, vol. 186 of Lecture Notes in Economics and Mathematical Systems, Springer, Berlin, Germany, 1981.

[30] M.-S. Kuo, G.-H. Tzeng, and W.-C. Huang, "Group decisionmaking based on concepts of ideal and anti-ideal points in a fuzzy environment," Mathematical and Computer Modelling, vol. 45, no. 3-4, pp. 324-339, 2007.

[31] F. E. Boran, S. Genã, and D. Akay, "Personnel selection based on intuitionistic fuzzy sets," Human Factors and Ergonomics in Manufacturing, vol. 21, no. 5, pp. 493-503, 2011.

[32] J. Tian, D. Yu, B. Yu, and S. Ma, "A fuzzy TOPSIS model via chisquare test for information source selection," Knowledge-Based Systems, vol. 37, pp. 515-527, 2012.

[33] H.-S. Shih, H.-J. Shyur, and E. S. Lee, "An extension of TOPSIS for group decision making," Mathematical and Computer Modelling, vol. 45, no. 7-8, pp. 801-813, 2007. 


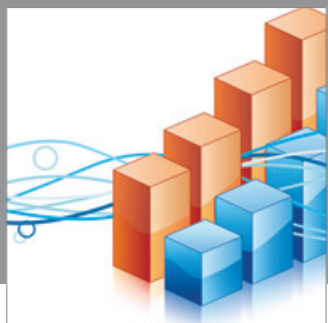

Advances in

Operations Research

mansans

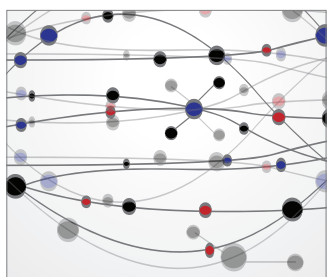

The Scientific World Journal
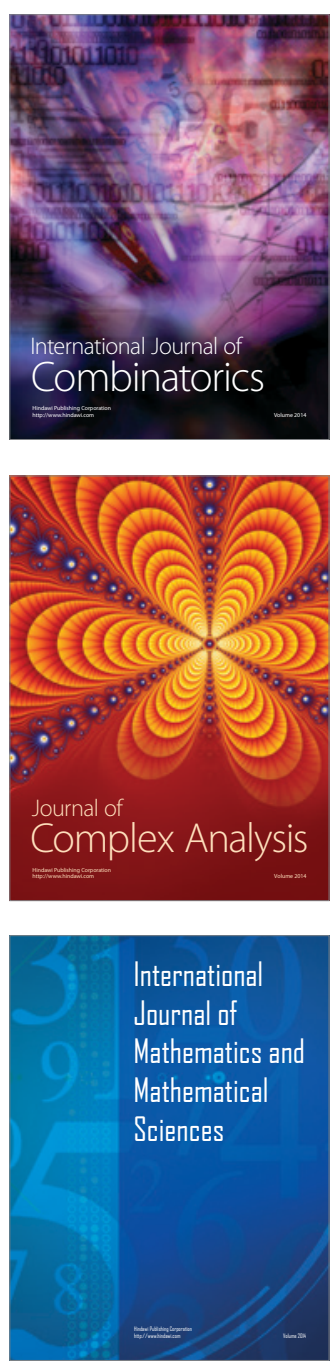
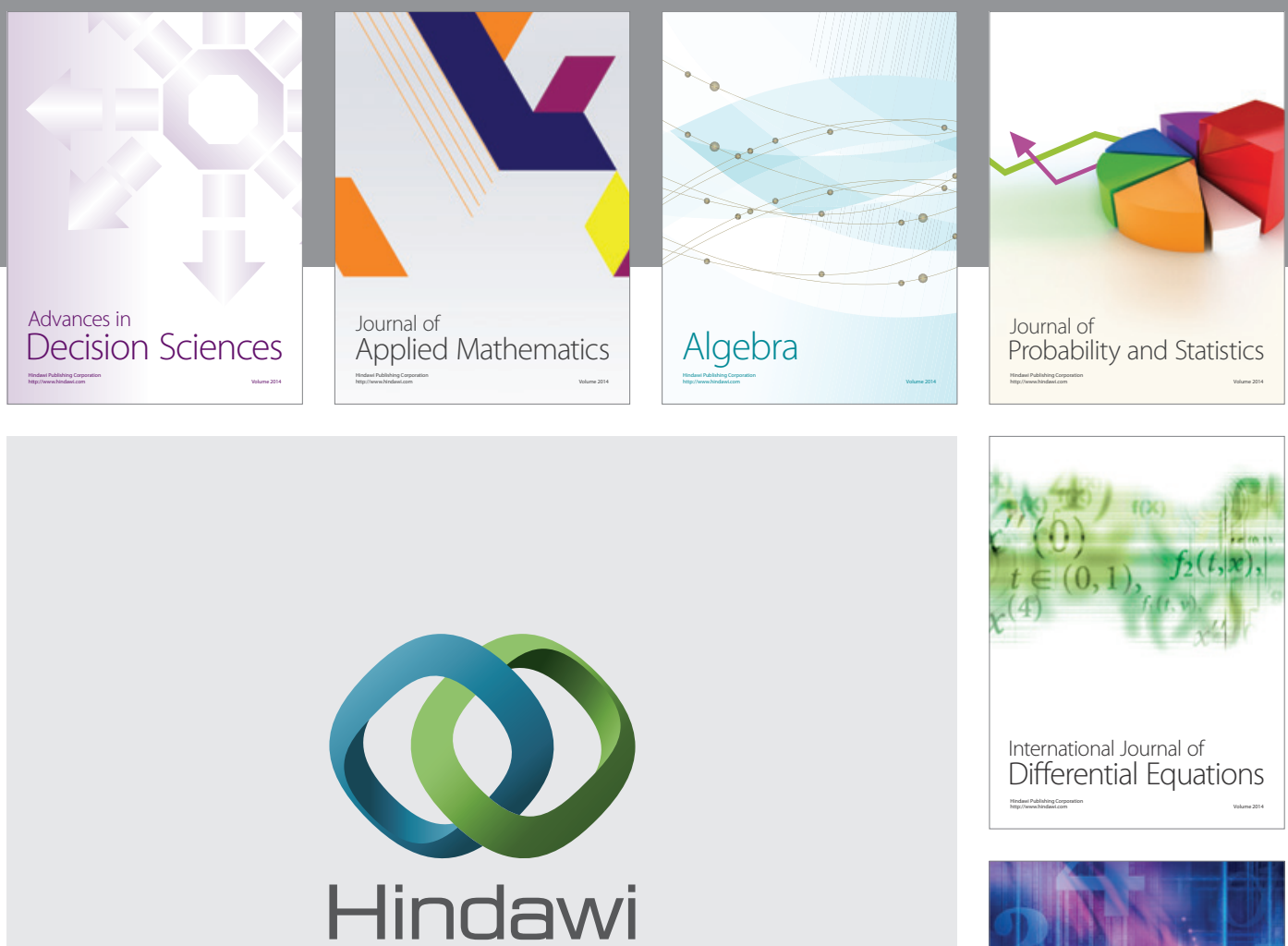

Submit your manuscripts at http://www.hindawi.com
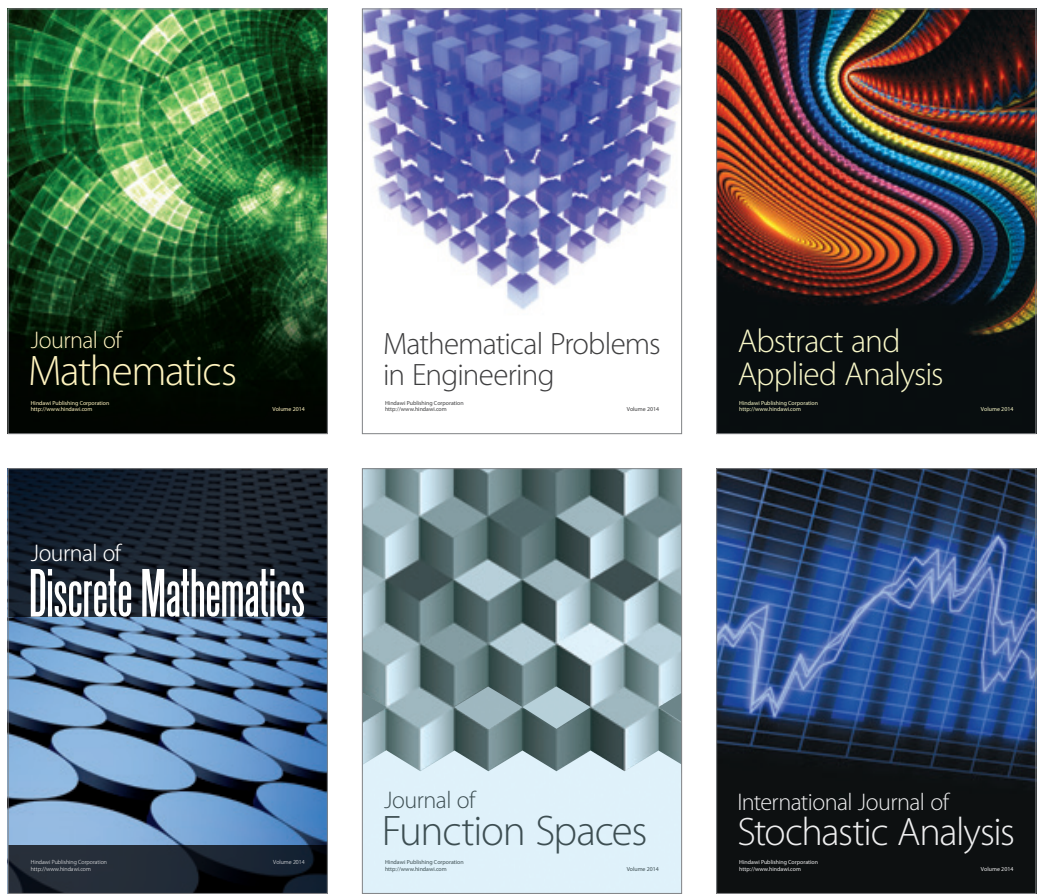

Journal of

Function Spaces

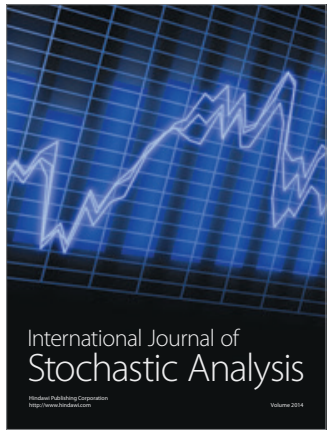

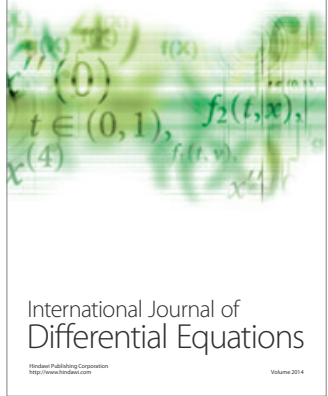
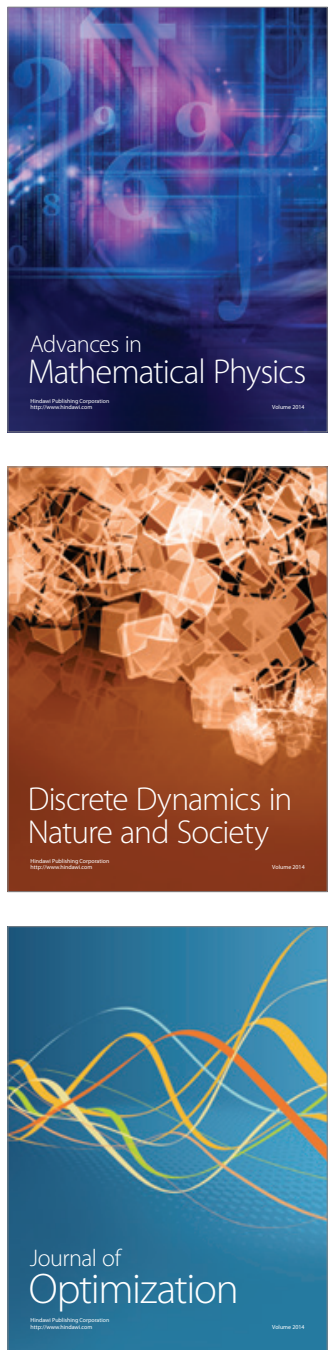